\title{
Dilatancy of granular materials in a strain space multiple mechanism model
}

\section{AUTHOR(S):}

lai, Susumu; Tobita, Tetsuo; Ozutsumi, Osamu; Ueda, Kyohei

\section{CITATION:}

lai, Susumu ...[et al]. Dilatancy of granular materials in a strain space multiple mechanism model. International Journal for Numerical and Analytical Methods in Geomechanics 2010, 35(3): 360-392

\section{ISSUE DATE:}

2010-03-11

URL:

http://hdl.handle.net/2433/197181

\section{RIGHT:}

This is the peer reviewed version of the following article: lai, S., Tobita, T., Ozutsumi, O. and Ueda, K. (2011), Dilatancy of granular materials in a strain space multiple mechanism model. Int. J. Numer. Anal. Meth. Geomech., 35: 360-392,

which has been published in final form at http://dx.doi.org/10.1002/nag.899; この論文は出版社版でありません。引用 の際には出版社版をご確認じ利用ください。; This is not the published version. Please cite only the published version. 
Short title: Dilatancy of granular materials

Dilatancy of granular materials in a strain space multiple mechanism model

$$
\text { Susumu Iai }{ }^{1} \text {, Tetsuo Tobita }{ }^{1} \text {, Osamu Ozutsumi }{ }^{2} \text {, and Kyohei Ueda }{ }^{1}
$$

${ }^{1}$ Disaster Prevention Research Institute, Kyoto University, Gokasho, Uji, Kyoto, 611-0011 Japan

${ }^{2}$ Meisosha Co., Sun Ikebukuro I-408, Ikebukuro 1-8-7, Toshima-ku, Tokyo 170-0014 Japan

Correspondence to:

Susumu Iai

Disaster Prevention Research Institute, Kyoto University, Gokasho, Uji, Kyoto, 611-0011 Japan e-mail: iai@geotech.dpri.kyoto-u.ac.jp

TEL 81-774-38-4090

FAX 81-774-38-4094 


\section{SUMMARY}

A granular material consists of an assemblage of particles with contacts newly formed or disappeared, changing the micromechanical structures during macroscopic deformation. These structures are idealized through a strain space multiple mechanism model as a two fold structure consisting of a multitude of virtual two dimensional mechanisms, each of which consists of a multitude of virtual simple shear mechanisms of one dimensional nature. In particular, a second order fabric tensor describes direct macroscopic stress strain relationship, and a fourth order fabric tensor describes incremental relationship. In this framework of modeling, the mechanism of interlocking defined as the energy less component of macroscopic strain provides an appropriate bridge between micromechanical and macroscopic dilative component of dilatancy. Another bridge for contractive component of dilatancy is provided through an obvious hypothesis on micromechanical counterparts being associated with virtual simple shear strain. It is also postulated that the dilatancy along the stress path beyond a line slightly above the phase transformation line is only due to the mechanism of interlocking and increment in dilatancy due to this interlocking eventually vanishes for a large shear strain. These classic postulates form the basis for formulating the dilatancy in the strain space multiple mechanism model. The performance of the proposed model is demonstrated through simulation of undrained behavior of sand under monotonic and cyclic loading.

Key words: constitutive equation; dilatancy; fabric tensor; granular material; micromechanics; steady state 


\section{INTRODUCTION}

The micromechanical structures of granular materials consist of an assemblage of particles with contacts newly formed or disappeared during macroscopic deformation. Among the various constitutive models proposed for granular materials, a strain space multiple mechanism model is one of the promising examples to relate these micromechanical structures to macroscopic deformation of granular materials [1-3]. The model consists of a multitude of simple shear mechanisms with each oriented in an arbitrary direction and can describe the behavior of granular materials under complicated loading paths, including the effect of rotation of principal stress axes $[1,4]$. The model has been implemented in a finite element program and used in the analysis of numerous problems in practice for evaluating seismic performance of geotechnical works including level ground, retaining structures, embankments, and underground structures [5-12].

In the previous studies along the line of a strain space multiple mechanism model, dilatancy was formulated either as a function of macroscopic cumulative shear work or with an assumption of stress-dilatancy relation in the microscopic level [1,2,13-15]. Although there is nothing wrong with those assumptions made as a part of numerical modeling, they seem to be made on an ad hoc basis and lack explicit discussions on the origin of dilatancy that reflects the micro/macro relationship on strain energy. A further discussion with respect to the origin of dilatancy may be beneficial to the better understanding of mechanics of granular materials along the line of thought in the strain space multiple mechanism. 
The objective of this study is to review the theoretical link between the micromechanical and macroscopic strain energy of granular materials and apply this link to the formulation of dilatancy in the strain space multiple mechanism model. The scope of this study is centered round the presentation of the formulation. The examples presented in this paper will be limited to those that are sufficient to confirm the fundamental applicability of the model. The specific advantages of the strain space multiple mechanism model will be discussed elsewhere.

\section{MULTIPLE MECHANISM IDEALIZED THROUGH FABRIC TENSORS}

\subsection{Concept and theoretical background}

Stress in granular materials as defined for continuum is given by a certain average of contact forces between the particles. In an assemblage of spherical particles, the contact force $\mathbf{P}$ can be partitioned into the direction of contact normal (or along the branch connecting the particle centers) $\mathbf{n}$ and tangential direction $\mathbf{t}$ as (see Figure 1)

$$
\mathbf{P}=F \mathbf{n}+S \mathbf{t}
$$

Macroscopic stress is given by taking an average over the contact forces within the representative volume element having volume $V$ as (e.g. Reference [16])

$$
\boldsymbol{\sigma}^{\prime}=\frac{1}{V} \sum l(F \mathbf{n} \otimes \mathbf{n}+S \mathbf{t} \otimes \mathbf{n})
$$

where $l$ denotes length of the branch.

Before taking the average over all the contacts of random orientation, a structure can be identified by systematically grouping the contacts according to the orientation. The first level of structures is identified by choosing those pairs of a contact force and a 
contact normal that are parallel to a plane specified by the local co-ordinates $\tilde{\mathbf{x}}, \tilde{\mathbf{y}}, \tilde{\mathbf{z}}$ with $\tilde{\mathbf{z}}$ axis normal to the plane as shown in Figure 2. Assemblage of those pairs on the plane constitutes a virtual two dimensional mechanism. The direction $\mathbf{n}$ within the plane is measured relative to the local coordinate $\tilde{\mathbf{x}}$ with an angle $\omega / 2$. The average of these two dimensional mechanisms over a surface of a unit sphere with respect to the solid angle $\Omega$ defines the strain space multiple mechanism model.

By systematically sorting out the isotropic and deviator components of the second order tensors in Equation (2) and by taking the number of summation to infinity, Equation (2) is rewritten as follows $[17,18]$ :

$$
\begin{gathered}
\boldsymbol{\sigma}^{\prime}=-p \mathbf{I}+\frac{1}{4 \pi} \iint\left(q_{F}\langle\mathbf{n} \otimes \mathbf{n}\rangle+q_{S}\langle\mathbf{t} \otimes \mathbf{n}\rangle\right) \mathrm{d} \omega \mathrm{d} \Omega \\
\langle\mathbf{n} \otimes \mathbf{n}\rangle=\mathbf{n} \otimes \mathbf{n}-\mathbf{t} \otimes \mathbf{t} \\
\langle\mathbf{t} \otimes \mathbf{n}\rangle=\mathbf{t} \otimes \mathbf{n}+\mathbf{n} \otimes \mathbf{t}
\end{gathered}
$$

where $p$ denotes effective confining pressure (compression positive), I denotes second order identity tensor, $q_{F}, q_{S}$ denote micromechanical stress contributions to macroscopic deviator stress due to normal and tangential components of contact forces.

Equation (3) represents the mechanisms with the combination of biaxial shear $\langle\mathbf{n} \otimes \mathbf{n}\rangle$ and the simple shear $\langle\mathbf{t} \otimes \mathbf{n}\rangle$. However, once they are idealized in terms of the second order tensors, they become indistinguishable except for the difference in the orientation with an angle of $\pi / 4$ as shown in Figure 3 [19]. Consequently, the term $\langle\mathbf{t} \otimes \mathbf{n}\rangle$, i.e. virtual simple shear mechanism, will be kept in use throughout this paper in order to maintain the consistency with those used in the previous papers $[1,2]$. Thus, Equation (3) is rewritten as [19-21]: 


$$
\boldsymbol{\sigma}^{\prime}=-p \mathbf{I}+\frac{1}{4 \pi} \iint q\langle\mathbf{t} \otimes \mathbf{n}\rangle \mathrm{d} \omega \mathrm{d} \Omega
$$

Equation (6) represents the two fold structure defined by the double integration to describe the macroscopic effective stress through a second order fabric tensor (for definition of fabric tensors, refer to e.g. [22]).

\subsection{Integrated form}

The integrated form of the constitutive equation, i.e. direct stress strain relationship, is derived by relating the macroscopic strain tensor $\boldsymbol{\varepsilon}$ to the macroscopic effective stress $\boldsymbol{\sigma}^{\prime}$ through the structure defined by Equation (6). The first step to derive this relationship is to define the volumetric strain $\varepsilon$ (extension positive) and the virtual simple shear strains $\gamma$ as the projections of the macroscopic strain field to the second order tensors representing volumetric and virtual simple shear mechanisms as follows:

$$
\begin{gathered}
\boldsymbol{\varepsilon}=\mathbf{I}: \boldsymbol{\varepsilon} \\
\gamma=\langle\mathbf{t} \otimes \mathbf{n}\rangle: \boldsymbol{\varepsilon}
\end{gathered}
$$

where the double dot symbol denotes double contraction. In order to take into account the effect of volumetric strain due to dilatancy $\varepsilon_{\mathrm{d}}$, effective volumetric strain $\varepsilon^{\prime}$ [23] is introduced by

$$
\varepsilon^{\prime}=\varepsilon-\varepsilon_{\mathrm{d}}
$$

where the rate of volumetric strain due to dilatancy is given by the projection of strain rate field to a second order tensor $\mathbf{I}_{\mathrm{d}}$ as

$$
\dot{\varepsilon}_{\mathrm{d}}=\mathbf{I}_{\mathrm{d}}: \dot{\boldsymbol{\varepsilon}}
$$

The scalar variables defined in Equations (8) and (9) as the projection of macroscopic 
strain field are used to define the isotropic stress $p$ and virtual simple shear stress $q$ in Equation (6) through path dependent functions as

$$
\begin{aligned}
& p=p\left(\varepsilon^{\prime}\right) \\
& q=q(\gamma)
\end{aligned}
$$

In the strain space multiple mechanism model, the virtual simple shear mechanism is formulated as a non-linear hysteretic function, where a back-bone curve is given by the following hyperbolic function [24];

$$
q(\gamma)=\frac{\gamma / \gamma_{\mathrm{v}}}{1+\left|\gamma / \gamma_{\mathrm{v}}\right|} q_{\mathrm{v}}
$$

The parameters $q_{\mathrm{v}}$ and $\gamma_{\mathrm{v}}$ defining the hyperbolic function are the shear strength and the reference strain of the virtual simple shear mechanism, respectively.

The strain space multiple mechanism model formulated with Equations (11) and (12) is the simplest in its kind to relate the macroscopic strain field to the macroscopic effective stress through a second order fabric tensor with strain induced anisotropy. This model fundamentally differs from a family of the multiple slip/multi-laminate models by Calladine [25,26] and Pande and Sharma [27] among others. As shown in Figure 4, the strain space multiple mechanism model or its family assumes that the particles in the granular materials moves, in average, consistently with the macroscopic strain field. This assumption allows defining the micromechanical strain as a projection of the macroscopic strain field through Equations (7) and (8). On the other hand, the family of the multiple slip/multi-laminate models assumes that the local strain is due to the multiple slip that can be significantly different from the macroscopic strain field. Although these two families of the models coincides with each other if the linear-elastic behavior is assumed for the micro (local) mechanism level, they are fundamentally 
different in its behavior if non-linear relationship or failure criteria is assumed for the micro (local) mechanism level.

As an example, two dimensional fabric mobilized by macroscopic simple shear in $x-y$ plane by the strain space multiple mechanism model is shown in Figure 5 . The fabric in strain space is defined in terms of $\gamma$, computed through Equation (8), and shown in Figure 5 (a). The fabric in stress space is defined in terms of $q$, given by a transformation of the strain space fabric through the non-linear path dependent function in Equation (12). In this example, the hyperbolic function in Equation (13) is used to obtain the stress space fabric shown in Figure 5 (b). The plus (+) and minus (-) symbols in Figure 5 indicate the signs of $\gamma$ and $q$. As shown in Figure 5, the strain space fabric evolves by increasing its magnitude in proportion to the macroscopic simple shear strain $\gamma_{x y}$ without changing its shape whereas the stress space fabric evolves from a slim shape of a four leaf clover into full bodied four leaves that cover an entire circle having a radius of the virtual shear strength $q_{\mathrm{v}}$. When the circle is fully covered, the material undergoes the macroscopic shear failure in the strain space multiple mechanism model.

In the mutliple slip/multi-laminate models, the orientation distributions of local stress is consistent with the macroscopic stress field because the local stress is given as a projection of macroscopic stress field. Because of the slip-mechanism assumed in the local plane, local shear strain tends to localize in the first slip plane that satisfies the failure criteria. An example may be found in reference [28] among others. Thus, the two families of the models are fundamentally different with each other.

\subsection{Inherent anisotropy}


The inherent anisotropy due to the texture of granular material can be readily introduced by generalizing the functions in Equations (11) and (12) being dependent on the orientation. By introducing the direction dependent parameters as

$$
\begin{aligned}
& p_{\mathrm{v}}=p_{\mathrm{v}}(\omega, \Omega) \\
& \varepsilon_{\mathrm{v}}=\varepsilon_{\mathrm{v}}(\omega, \Omega) \\
& q_{\mathrm{v}}=q_{\mathrm{v}}(\omega, \Omega) \\
& \gamma_{\mathrm{v}}=\gamma_{\mathrm{v}}(\omega, \Omega)
\end{aligned}
$$

the constitutive equation with inherent anisotropy may be written as follows:

$$
\begin{gathered}
\boldsymbol{\sigma}^{\prime}=-\frac{1}{4 \pi} \iint p^{*}|\mathbf{n} \otimes \mathbf{n}| \mathrm{d} \omega \mathrm{d} \Omega+\frac{1}{4 \pi} \iint q\langle\mathbf{t} \otimes \mathbf{n}\rangle \mathrm{d} \omega \mathrm{d} \Omega \\
|\mathbf{n} \otimes \mathbf{n}|=\mathbf{n} \otimes \mathbf{n}+\mathbf{t} \otimes \mathbf{t} \\
p^{*}=\bar{p}^{*}\left(\varepsilon^{\prime} / \varepsilon_{\mathrm{v}}\right) p_{\mathrm{v}} \\
q=\bar{q}\left(\gamma / \gamma_{\mathrm{v}}\right) q_{\mathrm{v}}
\end{gathered}
$$

where $\bar{p}^{*}$ and $\bar{q}$ are path dependent non-linear functions without direction dependency. This formulation is general enough to allow introduction of the higher modes of anisotropy in addition to the fundamental mode.

For example, the transformation process from the strain space fabric $\gamma$ to the stress space fabric $q$ through Equation (21) takes the following steps: (a) $\gamma \mapsto \gamma / \gamma_{\mathrm{v}}$, (b) $\gamma / \gamma_{\mathrm{v}} \mapsto q / q_{\mathrm{v}}$ and (c) $q / q_{\mathrm{v}} \mapsto q$. The inherent anisotropy comes into effect in the steps (a) and (c) that are linear transformation. The inherent anisotropy does not affect the transformation process in the step (b) that is essential for describing the non-linear evolution of stress space fabric such as shown in Figure 5 through Equation (13). Thus 
the generalization to include the inherent anisotropy for describing the texture of the material in various order of complexity is, in principle, simple in the strain space multiple mechanism model.

\subsection{Incremental form}

Incremental form of the constitutive equation is derived by taking the time derivative of both sides of Equation (6) as

$$
\begin{gathered}
\dot{\boldsymbol{\sigma}}^{\prime}=-\dot{p} \mathbf{I}+\frac{1}{4 \pi} \iint \dot{q}\langle\mathbf{t} \otimes \mathbf{n}\rangle \mathrm{d} \omega \mathrm{d} \Omega \\
\dot{p}=\frac{\mathrm{d} p}{\mathrm{~d} \varepsilon^{\prime}} \dot{\varepsilon}^{\prime} \\
\dot{q}=\frac{\partial q}{\partial \gamma} \dot{\gamma}
\end{gathered}
$$

Substitution of Equations (7) through (10) yields the incremental form of the constitutive equation as follows:

$$
\begin{gathered}
\dot{\boldsymbol{\sigma}}^{\prime}=\square: \dot{\boldsymbol{\varepsilon}} \\
\square=K_{\mathrm{L} / \mathrm{I}} \mathbf{I} \otimes \mathbf{I}+\frac{1}{4 \pi} \iint G_{\mathrm{L} / \mathrm{U}}\langle\mathbf{t} \otimes \mathbf{n}\rangle \otimes\langle\mathbf{t} \otimes \mathbf{n}\rangle \mathrm{d} \omega \mathrm{d} \Omega-K_{\mathrm{L} / \mathrm{U}} \mathbf{I} \otimes \mathbf{I}_{\mathrm{d}}
\end{gathered}
$$

where the terms of the tangential stiffness

$$
\begin{gathered}
K_{\mathrm{L} / \mathrm{U}}=-\frac{\mathrm{d} p}{\mathrm{~d} \varepsilon^{\prime}} \\
G_{\mathrm{L} / \mathrm{U}}=\frac{\partial q}{\partial \gamma}
\end{gathered}
$$

are given as path dependent functions and defined depending on the sign of strain rate component by 


$$
\begin{aligned}
& K_{\mathrm{L} / \mathrm{U}}=\left\{\begin{array}{llc}
K_{\mathrm{L}} & \text { if } \dot{\varepsilon}^{\prime} \geq 0 & \text { (loading/neutral) } \\
K_{\mathrm{U}} & \text { if } \dot{\varepsilon}^{\prime}<0 & \text { (unloading) }
\end{array}\right.
\end{aligned}
$$

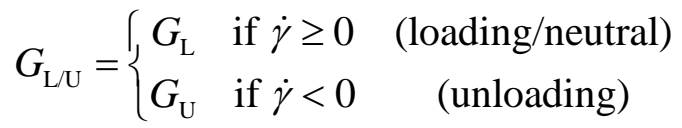

Thus the incremental form of the strain space multiple mechanism model is given through a fourth order fabric tensor $\square$ with strain induced anisotropy. The first and second terms of Equation (26) represent volumetric and deviator relationships, being major symmetric. The third term represents the effect of dilatancy that is formulated as a coupling between the volumetric and deviator mechanisms, being major unsymmetric.

\section{GENERALIZED MULTIPLE MECHANISM}

\subsection{Integrated form}

The fundamental form of the constitutive equations reviewed above can be generalized as follows. With holding Equations (6) through (8) being the same, dilatancy in Equation (9) is decomposed into contractive component $\varepsilon_{d}^{c}$ and dilative component $\varepsilon_{d}^{d}$ as

$$
\varepsilon_{\mathrm{d}}=\varepsilon_{\mathrm{d}}^{\mathrm{c}}+\varepsilon_{\mathrm{d}}^{\mathrm{d}}
$$

The physical motivation for such split may be given as follows. One motivation is due to the dilatancy at the minimum void ratio of sand (i.e. in a fully packed state). At this void ratio, contraction due to dilatancy is not possible so that the mechanism of dilatancy produces only the dilative volumetric strain. This conceptual experiment motivates for the concept of the dilative component of dilatancy to be defined as a mechanism different from the mechanism of dilatancy for producing contractive 
volumetric strain. The other motivation is due to the dilatancy during cyclic shear in the laboratory at the void ratio larger than the minimum. During the cyclic shear, accumulated volumetric strain is contractive as if sand accumulates non reversible damage. This evidence motives for the concept of the contractive component of dilatancy to be defined as a mechanism different from the mechanism of dilatancy for producing dilative volumetric strain. The underlying concept described above may be illustrated as shown in Figure 6. This concept was originally proposed by Fukutake [29] in the context of a bowl shaped dilative component of dilatancy.

By using the contractive component, virtual effective volumetric strain $\varepsilon^{\prime \prime}$ is defined as follows:

$$
\varepsilon^{\prime \prime}=\varepsilon-\varepsilon_{\mathrm{d}}^{\mathrm{c}}
$$

The virtual effective volumetric strain $\varepsilon "$ is the component of the effective volumetric strain without the effect of dilative component of dilatancy and will be used to represent the degree or state of liquefaction.

With holding Equation (11) being the same, Equation (12) is generalized in order take into account the various failure criteria defined for three dimensional model [19], and the effects of effective stress and state of liquefaction by introducing additional variables as

$$
q=q\left(\gamma, \varepsilon^{\prime}, \varepsilon^{\prime \prime}, J_{2}, \theta\right)
$$

where $J_{2}$ and $\theta$ denote the second invariant of stress tensor and Lode angle.

\subsection{Incremental form}

Derivation of the incremental form is given step-by-step as follow. First of all, the time 
derivative of both sides of Equation (33) yields

$$
\dot{q}=\frac{\partial q}{\partial \gamma} \dot{\gamma}+\frac{\partial q}{\partial \varepsilon^{\prime}} \dot{\varepsilon}^{\prime}+\frac{\partial q}{\partial \varepsilon^{\prime \prime}} \dot{\varepsilon}^{\prime \prime}+\frac{\partial q}{\partial J_{2}} \dot{J}_{2}+\frac{\partial q}{\partial \theta} \dot{\theta}
$$

Volumetric strain rate due to dilatancy is given through the derivation described later as follows:

$$
\begin{gathered}
\dot{\varepsilon}_{\mathrm{d}}=\mathbf{I}_{\mathrm{d}}: \dot{\boldsymbol{\varepsilon}}+\mathbf{J}_{\mathrm{d}}: \dot{\boldsymbol{\sigma}}^{\prime} \\
\dot{\varepsilon}_{\mathrm{d}}^{\mathrm{c}}=\mathbf{I}_{\mathrm{d}}^{\mathrm{c}}: \dot{\boldsymbol{\varepsilon}}
\end{gathered}
$$

Consequently, the strain rates appearing in the right side of Equation (34) are written as

$$
\begin{gathered}
\dot{\gamma}=\langle\mathbf{t} \otimes \mathbf{n}\rangle: \dot{\boldsymbol{\varepsilon}} \\
\dot{\varepsilon}^{\prime}=\left(\mathbf{I}-\mathbf{I}_{\mathrm{d}}\right): \dot{\boldsymbol{\varepsilon}}-\mathbf{J}_{\mathrm{d}}: \dot{\boldsymbol{\sigma}}^{\prime} \\
\dot{\varepsilon}^{\prime \prime}=\left(\mathbf{I}-\mathbf{I}_{\mathrm{d}}^{\mathrm{c}}\right): \dot{\boldsymbol{\varepsilon}}
\end{gathered}
$$

Similarly, the stress rates appearing in the right side of Equation (34) are written as

$$
\begin{aligned}
& \dot{J}_{2}=\frac{\partial J_{2}}{\partial \boldsymbol{\sigma}^{\prime}}: \dot{\boldsymbol{\sigma}}^{\prime} \\
& \dot{\theta}=\frac{\partial \theta}{\partial \boldsymbol{\sigma}^{\prime}}: \dot{\boldsymbol{\sigma}}^{\prime}
\end{aligned}
$$

Substitution of Equations (37) through (41) into Equations (23) and (34) yields

$$
\begin{gathered}
\dot{p}=\frac{\mathrm{d} p}{\mathrm{~d} \varepsilon^{\prime}}\left(\mathbf{I}-\mathbf{I}_{\mathrm{d}}\right): \dot{\boldsymbol{\varepsilon}}-\frac{\mathrm{d} p}{\mathrm{~d} \varepsilon^{\prime}} \mathbf{J}_{\mathrm{d}}: \dot{\boldsymbol{\sigma}}^{\prime} \\
\dot{q}=\frac{\partial q}{\partial \gamma}\langle\mathbf{t} \otimes \mathbf{n}\rangle: \dot{\boldsymbol{\varepsilon}}+\frac{\partial q}{\partial \varepsilon^{\prime}}\left(\mathbf{I}-\mathbf{I}_{\mathrm{d}}\right): \dot{\boldsymbol{\varepsilon}}-\frac{\partial q}{\partial \varepsilon^{\prime}} \mathbf{J}_{\mathrm{d}}: \dot{\boldsymbol{\sigma}}^{\prime} \\
+\frac{\partial q}{\partial \varepsilon^{\prime \prime}}\left(\mathbf{I}-\mathbf{I}_{\mathrm{d}}^{\mathrm{c}}\right): \dot{\boldsymbol{\varepsilon}}+\left(\frac{\partial q}{\partial J_{2}} \frac{\partial J_{2}}{\partial \boldsymbol{\sigma}^{\prime}}+\frac{\partial q}{\partial \theta} \frac{\partial \theta}{\partial \boldsymbol{\sigma}^{\prime}}\right): \dot{\boldsymbol{\sigma}}^{\prime}
\end{gathered}
$$

By substituting these equations into Equation (22), the generalized incremental constitutive equation is given as follows: 


$$
\begin{gathered}
\dot{\boldsymbol{\sigma}}^{\prime}=\square: \dot{\boldsymbol{\varepsilon}}+\square: \dot{\boldsymbol{\sigma}}^{\prime} \\
\square=K_{\mathrm{L} / \mathrm{U}} \mathbf{I} \otimes \mathbf{I}+\frac{1}{4 \pi} \iint G_{\mathrm{L} / \mathrm{U}}\langle\mathbf{t} \otimes \mathbf{n}\rangle \otimes\langle\mathbf{t} \otimes \mathbf{n}\rangle \mathrm{d} \omega \mathrm{d} \Omega-K_{\mathrm{L} / \mathrm{U}} \mathbf{I} \otimes \mathbf{I}_{\mathrm{d}} \\
+\frac{1}{4 \pi} \iint H\langle\mathbf{t} \otimes \mathbf{n}\rangle \mathrm{d} \omega \mathrm{d} \Omega \otimes\left(\mathbf{I}-\mathbf{I}_{\mathrm{d}}\right)+\frac{1}{4 \pi} \iint L\langle\mathbf{t} \otimes \mathbf{n}\rangle \mathrm{d} \omega \mathrm{d} \Omega \otimes\left(\mathbf{I}-\mathbf{I}_{\mathrm{d}}^{\mathrm{c}}\right) \\
\square=-K_{\mathrm{L} / \mathrm{U}} \mathbf{I} \otimes \mathbf{J}_{\mathrm{d}}-\frac{1}{4 \pi} \iint H\langle\mathbf{t} \otimes \mathbf{n}\rangle \mathrm{d} \omega \mathrm{d} \Omega \otimes \mathbf{J}_{\mathrm{d}}+\frac{1}{4 \pi} \iint\langle\mathbf{t} \otimes \mathbf{n}\rangle \otimes \mathbf{J}_{q} \mathrm{~d} \omega \mathrm{d} \Omega
\end{gathered}
$$

where

$$
\begin{gathered}
H=\frac{\partial q}{\partial \varepsilon^{\prime}} \\
L=\frac{\partial q}{\partial \varepsilon^{\prime \prime}} \\
\mathbf{J}_{q}=\frac{\partial q}{\partial J_{2}} \frac{\partial J_{2}}{\partial \boldsymbol{\sigma}^{\prime}}+\frac{\partial q}{\partial \theta} \frac{\partial \theta}{\partial \boldsymbol{\sigma}^{\prime}}
\end{gathered}
$$

The first through third terms in the right side of Equation (45) are the same as those appearing in the fundamental form given in Equation (26). The generalized form includes the fourth term representing the dependency of shear mechanism on effective stress and the fifth term on the state of liquefaction. The third through fifth terms represent coupling between the volumetric and shear mechanisms, being major unsymmetric. Equation (46) also includes the effects of the second invariant of stress tensor $J_{2}$ and Lode angle $\theta$ on the shear and dilatancy as represented by the fourth order fabric tensor $\square$.

\section{STRAIN ENERGY AND DILATANCY}

\subsection{Micro- and macroscopic strain energy}


As a prelude to the formulation of dilatancy in the strain space multiple mechanism model, relationship between the microscopic and macroscopic strain energies are identified as follows. First of all, the rate of strain energy in the strain space multiple mechanism model $\dot{W}$ is computed through Equations (6), (7) and (8) as

$$
\dot{W}=\boldsymbol{\sigma}^{\prime}: \dot{\boldsymbol{\varepsilon}}=\left(-p \mathbf{I}+\frac{1}{4 \pi} \iint q\langle\mathbf{t} \otimes \mathbf{n}\rangle \mathrm{d} \omega \mathrm{d} \Omega\right): \dot{\boldsymbol{\varepsilon}}=-p \dot{\varepsilon}+\frac{1}{4 \pi} \iint q \dot{\gamma} \mathrm{d} \omega \mathrm{d} \Omega
$$

Equation (50) can be rewritten as

$$
\dot{W}=\boldsymbol{\sigma}^{\prime}: \dot{\boldsymbol{\varepsilon}}=\dot{W}_{p}+\frac{1}{4 \pi} \iint \dot{W}_{q \mathrm{v}} \mathrm{d} \omega \mathrm{d} \Omega
$$

where

$$
\begin{gathered}
\dot{W}_{p}=-p \dot{\varepsilon} \\
\dot{W}_{q \mathrm{v}}=q \dot{\gamma}
\end{gathered}
$$

Equation (51) defines the fundamental relationship between the macroscopic strain energy rate $\dot{W}=\sigma^{\prime}: \dot{\boldsymbol{\varepsilon}}$ and the microscopic strain energy rate $\dot{W}_{q v}=q \dot{\gamma}$ given by the individual virtual simple shear mechanism that constitutes the strain space multiple mechanism model. This relationship will play a major role in formulating dilatancy as described later. This relationship plays another major role in formulating hysteretic damping during cyclic shear but this part of the discussions, though important, will be left to the references $[30,31]$ in order to streamline the paper.

Based on Equations (9) and (31), the volumetric strain rate is divided into three components, i.e. component due to isotropic stress change, and contractive and dilative components of dilatancy as follows:

$$
\dot{\varepsilon}=\dot{\varepsilon}^{\prime}+\dot{\varepsilon}_{\mathrm{d}}^{\mathrm{c}}+\dot{\varepsilon}_{\mathrm{d}}^{\mathrm{d}}
$$

Substitution of Equation (54) into Equation (51) yields 


$$
\dot{W}=\boldsymbol{\sigma}^{\prime}: \dot{\boldsymbol{\varepsilon}}=\dot{W}_{p}^{\prime}+\dot{W}_{p}^{\mathrm{dc}}+\dot{W}_{p}^{\mathrm{dd}}+\frac{1}{4 \pi} \iint \dot{W}_{q \mathrm{v}} \mathrm{d} \omega \mathrm{d} \Omega
$$

where

$$
\begin{gathered}
\dot{W}_{p}{ }^{\prime}=-p \dot{\varepsilon}^{\prime} \\
\dot{W}_{p}^{\mathrm{dc}}=-p \dot{\varepsilon}_{\mathrm{d}}^{\mathrm{c}} \\
\dot{W}_{p}^{\mathrm{dd}}=-p \dot{\varepsilon}_{\mathrm{d}}^{\mathrm{d}}
\end{gathered}
$$

\subsection{Dilative component of dilatancy}

Following the classic postulate of interlocking that is defined as the energy less component of deformation by Taylor [32], a hypothesis is made in this study that a fraction of the shear strain energy is consumed in the mechanism of interlocking as

$$
\dot{W}_{p}^{\mathrm{dd}}+\frac{1}{4 \pi} \iint \dot{W}_{q \mathrm{v}}^{\mathrm{dd}} \mathrm{d} \omega \mathrm{d} \Omega=0
$$

where, by using the parameter $r_{\varepsilon_{\mathrm{d}}}$, the fraction of the shear strain energy rate is given by

$$
\dot{W}_{q \mathrm{v}}^{\mathrm{dd}}=r_{\varepsilon_{\mathrm{d}}} \dot{W}_{q \mathrm{v}}=r_{\varepsilon_{\mathrm{d}}} q \dot{\gamma}
$$

The strain energy relation hypothesized in the original Cam-Clay model $[33,34]$ may be interpreted as a special case where the parameter $r_{\varepsilon_{\mathrm{d}}}$ is reduced to unity so that the strain energy is only due to the contractive component of dilatancy $\dot{W}_{p}^{\mathrm{dc}}=-p \dot{\varepsilon}_{\mathrm{d}}^{\mathrm{c}}$ defined in Equation (57).

In this study, it is also postulated that the shear stress contribution $q$ to the interlocking in Equation (60) refers to the backbone curve in Equation (13). This postulate is made in order to avoid accumulation of dilative component of dilatancy 
during cyclic loading and thus simplifies the formulation of dilatancy. A conceptual background to the mechanism of interlocking may well be given by imagining a fictitious structure consisting of void (to be called void skeleton) that supplements the soil skeleton to form a specific continuum to idealize the behavior of granular materials with an assumption that the work done by the void skeleton is none [35].

Based on these postulates together with Equations (53) and (58), dilative component of dilatancy is given by

$$
\dot{\varepsilon}_{\mathrm{d}}^{\mathrm{d}}=\frac{1}{4 \pi} r_{\varepsilon_{\mathrm{d}}} \iint \frac{\gamma / \gamma_{\mathrm{v}}}{1+\left|\gamma / \gamma_{\mathrm{v}}\right|} \mathrm{M}_{\mathrm{fv}} \dot{\gamma} \mathrm{d} \omega \mathrm{d} \Omega
$$

where

$$
\mathrm{M}_{\mathrm{fv}}=\frac{q_{\mathrm{v}}}{p}
$$

is nothing but a slope of the shear failure line defined for the virtual simple shear mechanism. Equation (61) is integrated with respect to $\gamma$ with the condition that $\varepsilon_{\mathrm{d}}^{\mathrm{d}}=0$ for $\gamma=0$ to derive an integrated form of dilative component of dilatancy as

$$
\varepsilon_{\mathrm{d}}^{\mathrm{d}}=\frac{1}{4 \pi} r_{\varepsilon_{\mathrm{d}}} \iint\left[\left|\frac{\gamma}{\gamma_{\mathrm{v}}}\right|-\ln \left(1+\left|\frac{\gamma}{\gamma_{\mathrm{v}}}\right|\right)\right]_{\mathrm{v}} \gamma_{\mathrm{fv}} \mathrm{d} \omega \mathrm{d} \Omega
$$

Thus the macroscopic dilative component of dilatancy is given from the micromechanical counterparts that are defined as a function of strain. Since stress is not directly involved in the mechanism formulated in this study, the dilatancy may well be interpreted as kinematic constraints due to the existence of particles and voids in granular materials.

Substitution of Equation (37) into Equation (61) yields the incremental relationship between the dilative component of dilatancy and the macroscopic strain through a 
second order fabric tensor $\mathbf{I}_{\mathrm{d}}^{\mathrm{d}}$ as follows:

$$
\begin{gathered}
\dot{\varepsilon}_{\mathrm{d}}^{\mathrm{d}}=\mathbf{I}_{\mathrm{d}}^{\mathrm{d}}: \dot{\boldsymbol{\varepsilon}} \\
\mathbf{I}_{\mathrm{d}}^{\mathrm{d}}=\frac{1}{4 \pi} r_{\varepsilon_{\mathrm{d}}} \iint \frac{\gamma / \gamma_{\mathrm{v}}}{1+\left|\gamma / \gamma_{\mathrm{v}}\right|} \mathrm{M}_{\mathrm{fv}}\langle\mathbf{t} \otimes \mathbf{n}\rangle \mathrm{d} \omega \mathrm{d} \Omega
\end{gathered}
$$

The dilative component of dilatancy during cyclic simple shear idealized through Equations (63) through (65) is shown in Figure 7. The curve forms a shape similar to the vertical cross section of a cocktail glass top and gradually approaches a straight line at both ends as the shear strain increases in its magnitude.

The formulation given above is the fundamental form of the dilative component of dilatancy. This formulation is extended for describing the steady state of soil by introducing an upper limit to the dilative component of dilatancy. In order to formulate this limit, Equation (63) may be extended by introducing a parameter $\gamma_{\text {vus }}$ representing an asymptote limit state as follows:

$$
\begin{gathered}
\varepsilon_{\mathrm{d}}^{\mathrm{d}}=\frac{1}{4 \pi} r_{\varepsilon_{\mathrm{d}}} \iint\left[\left|\frac{\tilde{\gamma}}{\gamma_{\mathrm{v}}}\right|-\ln \left(1+\mid \frac{\tilde{\gamma}}{\gamma_{\mathrm{v}}}\right)\right]_{]} \gamma_{\mathrm{v}} \mathrm{M}_{\mathrm{fv}} \mathrm{d} \omega \mathrm{d} \Omega \\
\left|\frac{\tilde{\gamma}}{\gamma_{\mathrm{v}}}\right|=\left(1-\exp \left(-\frac{|\gamma|}{\gamma_{\text {vus }}}\right)\right) \frac{\gamma_{\text {vus }}}{\gamma_{\mathrm{v}}}
\end{gathered}
$$

The incremental form is given by replacing Equation (65) with

$$
\mathbf{I}_{\mathrm{d}}^{\mathrm{d}}=\frac{1}{4 \pi} r_{\varepsilon_{\mathrm{d}}} \iint \frac{\tilde{\gamma} / \gamma_{\mathrm{v}}}{1+\left|\tilde{\gamma} / \gamma_{\mathrm{v}}\right|} \operatorname{sgn}(\gamma) \exp \left(-\frac{|\gamma|}{\gamma_{\text {vus }}}\right) \mathrm{M}_{\mathrm{fv}}\langle\mathbf{t} \otimes \mathbf{n}\rangle \mathrm{d} \omega \mathrm{d} \Omega
$$

Later on in this paper, the formulation is further generalized to take into account the dependency of $\gamma_{\mathrm{v}}$ on the virtual effective strain $\varepsilon$ ".

\subsection{Contractive component of dilatancy}


The contractive component of dilatancy is given by the simple hypothesis in that the macroscopic component is given from those of microscopic counterparts associated with the virtual simple shear strain rate as follows:

$$
\dot{\varepsilon}_{\mathrm{d}}^{\mathrm{c}}=-\frac{1}{4 \pi} \iint \mathrm{M}_{\mathrm{v}}\left|\dot{\gamma}_{\mathrm{p}}\right| \mathrm{d} \omega \mathrm{d} \Omega
$$

where $\gamma_{\mathrm{p}}$ denotes irreversible (plastic) portion of virtual simple shear strain, being defined by

$$
\dot{\gamma}_{\mathrm{p}}=\dot{\gamma}-c_{1} \dot{\gamma}_{\mathrm{e}}
$$

A parameter $c_{1}$ controls the elastic range and, if $\dot{\gamma} \leq c_{1} \dot{\gamma}_{\mathrm{e}}$, then $\dot{\gamma}_{\mathrm{p}}=0$. By applying Equations (13) and (28) for a small strain range, Equation (70) is rewritten as

$$
\dot{\gamma}_{\mathrm{p}}=\left(1-c_{1}\left(\frac{G_{\mathrm{L} / \mathrm{U}}}{G_{\mathrm{L} 0}}\right)\right) \dot{\gamma}
$$

where, if $1<c_{1}\left(\frac{G_{\mathrm{L} / \mathrm{U}}}{G_{\mathrm{L} 0}}\right)$, then $\dot{\gamma}_{\mathrm{p}}=0$ and

$$
G_{\mathrm{L} 0}=\frac{q_{\mathrm{v}}}{\gamma_{\mathrm{v}}}
$$

Consequently, the contractive component of dilatancy is given by

$$
\dot{\varepsilon}_{\mathrm{d}}^{\mathrm{c}}=-\frac{1}{4 \pi} \iint \mathrm{M}_{\mathrm{v}}\left(1-c_{1}\left(\frac{G_{\mathrm{L} / \mathrm{U}}}{G_{\mathrm{L} 0}}\right)\right)|\dot{\gamma}| \mathrm{d} \omega \mathrm{d} \Omega
$$

and, if $1<c_{1}\left(\frac{G_{\mathrm{L} / \mathrm{U}}}{G_{\mathrm{L} 0}}\right)$, that component of the term in integral vanishes.

Thus, the contractive component of dilatancy is formulated through another second order fabric tensor $\mathbf{I}_{\mathrm{d}}^{\mathrm{c}}$ as follows:

$$
\dot{\varepsilon}_{\mathrm{d}}^{\mathrm{c}}=\mathbf{I}_{\mathrm{d}}^{\mathrm{c}}: \dot{\boldsymbol{\varepsilon}}
$$




$$
\mathbf{I}_{\mathrm{d}}^{\mathrm{c}}=-\frac{1}{4 \pi} \iint \mathrm{M}_{\mathrm{v}}\left(1-c_{1}\left(\frac{G_{\mathrm{L} / \mathrm{U}}}{G_{\mathrm{L} 0}}\right)\right)|\langle\mathbf{t} \otimes \mathbf{n}\rangle|^{*} \mathrm{~d} \omega \mathrm{d} \Omega
$$

where, if $\langle\mathbf{t} \otimes \mathbf{n}\rangle: \dot{\boldsymbol{\varepsilon}} \geq 0$, then

$$
|\langle\mathbf{t} \otimes \mathbf{n}\rangle|^{*}=\langle\mathbf{t} \otimes \mathbf{n}\rangle
$$

and , if $\langle\mathbf{t} \otimes \mathbf{n}\rangle: \dot{\boldsymbol{\varepsilon}}<0$, then

$$
|\langle\mathbf{t} \otimes \mathbf{n}\rangle|^{*}=-\langle\mathbf{t} \otimes \mathbf{n}\rangle
$$

The formulation given above is the fundamental form of the contractive component of dilatancy. Later on in this paper, the formulation will be generalized by introducing parameters for controlling the rate and the dependency on the virtual effective strain $\varepsilon$ ". It will also be postulated that no increase is allowed for contractive component of dilatancy along the stress path beyond a limiting line that is defined between the failure line and the phase transformation line [36].

Figure 8 shows the position of this limiting line relative to the phase transformation and failure lines in a stress plane defined by effective confining pressure $p$ and shear stress $\tau=\sqrt{J_{2}} \cos \theta$. In particular, the zone above the limiting line indicated by hatching in Figure 8 allows only dilative component of dilatancy due to interlocking; the steady state cannot be reached without going through this zone to the failure line. This line of thought is getting along the modern interpretation of strain energy [37]. However, this type of modeling is general enough to allow variation depending on the type and state of soil. For example, the failure and limiting lines are allowed to coincide with the phase transformation line. In this case, the stress path similar to that given by the original Cam-Clay model $[33,34]$ is realized when the steady state (i.e. the critical 
state) is approached from the wet side.

\section{MODEL PARAMETERS}

\subsection{Parameters for volumetric mechanism}

In order to study the applicability of the dilatancy model proposed in Chapter 4, the model is applied for two dimensional analysis of sand by reducing the double integration such as shown in Equation (6) to single integration with respect to $\omega$. For the sake of simplicity, the texture of the material is assumed to be isotropic. Details, including the vector-matrix representation used for numerical analysis, may be found in the previous studies [2,38]. The model parameters and the functions required to complete the description of the constitutive equation are as follows.

By assigning the reference confining pressure $p_{\mathrm{a}}$, bulk moduli in Equation (29) are given, for normal consolidation, by

$$
K_{\mathrm{L}}=K_{\mathrm{La}}\left(\frac{p}{p_{\mathrm{a}}}\right)^{n_{K}}
$$

and, for over consolidation, by

$$
K_{\mathrm{U}}=K_{\text {Ua }}\left(\frac{p}{p_{\mathrm{a}}}\right)^{n_{K}}
$$

In the analysis of liquefaction and other undrained or partially drained behavior of sand, this is extended, by referring to the initial confining pressure $p_{0}$, as

$$
K_{\mathrm{L} / \mathrm{U}}=r_{K} K_{\mathrm{U} 0}\left(\frac{p}{p_{0}}\right)^{l_{K}}
$$

The parameter $r_{K}$ is introduced to account for the difference in the bulk modulus for 
drained and undrained conditions. The power index $l_{K}$ is allowed to take the value larger than or equal to unity in order to simulate the liquefaction phenomena where the contractive component of dilatancy reaches the volumetric strain, ranging from one to five percent, that exceeds the volumetric strain induced by normal consolidation preceding the undrained cyclic loading [39]. Integrated form of Equation (80) is given, for $l_{K} \neq 1$, by

$$
\begin{gathered}
p=p_{0}(\eta+1)^{\frac{1}{1-l_{\mathrm{K}}}} \\
\eta=-\left(1-l_{K}\right)\left(\varepsilon^{\prime}-\varepsilon_{0}{ }^{\prime}\right) / \varepsilon_{\mathrm{m} 0}
\end{gathered}
$$

and, for $l_{K}=1$, by

$$
\begin{gathered}
p=p_{0} \exp \eta \\
\eta=-\left(\varepsilon^{\prime}-\varepsilon_{0}{ }^{\prime}\right) / \varepsilon_{\mathrm{m} 0}
\end{gathered}
$$

where the term

$$
\varepsilon_{\mathrm{m} 0}=p_{0} /\left(r_{K} K_{\mathrm{U} 0}\right)
$$

may well be called the reference volumetric strain from the analogy to the reference strain defined for shear.

\subsection{Parameters for shear mechanism}

The parameters $q_{\mathrm{v}}$ and $\gamma_{\mathrm{v}}$ defining the shear mechanism such as defined in Equation (13) are given from the macroscopic shear strength $\tau_{\mathrm{m}}$ and elastic shear modulus $G_{\mathrm{m}}$ as follows [2]:

$$
q_{\mathrm{v}}=\frac{\tau_{\mathrm{m}}}{\sum_{i=1}^{I} \sin \omega_{i} \Delta \omega} \approx \frac{\tau_{\mathrm{m}}}{2}
$$




$$
\gamma_{\mathrm{v}}=\left(\frac{\sum_{i=1}^{I} \sin ^{2} \omega_{i} \Delta \omega}{\sum_{i=1}^{I} \sin \omega_{i} \Delta \omega}\right) \gamma_{\mathrm{m}} \approx \frac{\pi}{4} \gamma_{\mathrm{m}}
$$

where $\gamma_{\mathrm{m}}=\tau_{\mathrm{m}} / G_{\mathrm{m}}$ is the reference strain often referred to in the discipline of soil dynamics and $\Delta \omega=\pi / I$ when the integration is replaced by summation in the numerical analysis. The slope of the failure line for virtual simple shear is given, by using Equations (62) and (86), as

$$
\mathrm{M}_{\mathrm{fv}}=\frac{\mathrm{M}_{\mathrm{f}}}{\sum_{i=1}^{I} \sin \omega_{i} \Delta \omega} \approx \frac{\mathrm{M}_{\mathrm{f}}}{2}
$$

The tangent stiffness used for the incremental relationship is given, from Equations (28) and (13), for the backbone curve as

$$
G_{\mathrm{L}}=\frac{\partial q}{\partial \gamma}=\frac{1}{\left(1+\left|\gamma / \gamma_{\mathrm{v}}\right|\right)^{2}} \frac{q_{\mathrm{v}}}{\gamma_{\mathrm{v}}}
$$

For the formulation of hysteretic behavior, refer to [30,31].

Confining pressure dependency is introduced to these parameters as follows:

$$
\begin{gathered}
\tau_{\mathrm{m}}=p \sin \phi_{\mathrm{f}}=\mathrm{M}_{\mathrm{f}} p \\
G_{\mathrm{m}}=G_{\mathrm{ma}}\left(\frac{p}{p_{\mathrm{a}}}\right)^{m_{G}} \\
\gamma_{\mathrm{m}}=\frac{\tau_{\mathrm{m}}}{G_{\mathrm{m}}}=m_{1} \frac{p}{G_{\mathrm{ma}}}\left(\frac{p}{p_{\mathrm{a}}}\right)^{-m_{G}}
\end{gathered}
$$

where $\phi_{\mathrm{f}}$ denotes internal friction angle to define the slope of failure line $\mathrm{M}_{\mathrm{f}}=\sin \phi_{\mathrm{f}}$.

In the analysis of liquefaction of sand, dependency on confining pressure and the state of liquefaction is introduced in reference to the previous study on modeling of 
cyclic mobility [2] and more recent laboratory studies under undrained condition [40] as follows:

for $S_{0}>S_{0 \mathrm{bd}}$ :

$$
\begin{gathered}
\tau_{\mathrm{m}}=\tau_{\mathrm{m} 0} S \\
G_{\mathrm{m}}=\tau_{\mathrm{m}} / \gamma_{\mathrm{m} 0} \\
\gamma_{\mathrm{m}}=\gamma_{\mathrm{m} 0}
\end{gathered}
$$

and, for $S_{0}<S_{0 \text { bd }}$ :

$$
\begin{gathered}
\tau_{\mathrm{m}}=\tau_{\mathrm{m} 0} S \\
G_{\mathrm{m}}=\tau_{\mathrm{m}} / \gamma_{\mathrm{m}} \\
\gamma_{\mathrm{m}}=\gamma_{\mathrm{m} 0} /\left(S_{0} / S_{0 \mathrm{bd}}\right)
\end{gathered}
$$

where

$$
S_{0 \mathrm{bd}}=1.0
$$

and the terms $\tau_{\mathrm{m} 0}$ and $\gamma_{\mathrm{m} 0}$ are defined through Equations (90) and (92) by referring to the initial confining pressure $p_{0}$ in the analysis of liquefaction. The state variable $S$ and the liquefaction front parameter $S_{0}$ appearing in Equations (93) through (98) are defined as follows:

$$
\begin{gathered}
S=p / p_{0} \\
S_{0}=\min \left(p^{\prime \prime} / p_{0}\right)
\end{gathered}
$$

where the lower limit for $S$ and $S_{0}$ is given by a parameter $S_{1}$ being a small positive number. The virtual effective stress $p^{\prime \prime}$ appearing in Equation (101) is defined similar to Equation (81) by replacing the effective volumetric strain $\varepsilon^{\prime}$ with the virtual 
effective volumetric strain $\varepsilon^{\prime \prime}$ as follows:

for $l_{K} \neq 1$ :

$$
\begin{gathered}
p^{\prime \prime}=p_{0}\left(\eta^{\prime \prime}+1\right)^{\frac{1}{1-l_{K}}} \\
\eta^{\prime \prime}=-\left(1-l_{K}\right)\left(\varepsilon^{\prime \prime}-\varepsilon_{0} "\right) / \varepsilon_{\mathrm{m} 0} "
\end{gathered}
$$

and, for $l_{K}=1$ :

$$
\begin{gathered}
p^{\prime \prime}=p_{0} \exp \eta " \\
\eta^{\prime \prime}=-\left(\varepsilon^{\prime \prime}-\varepsilon_{0}{ }^{\prime \prime}\right) / \varepsilon_{\mathrm{m} 0} "
\end{gathered}
$$

where the term

$$
\varepsilon_{\mathrm{m} 0} "=p_{0} /\left(r_{K} " K_{\mathrm{U} 0}\right)
$$

is defined by using a parameter $r_{K}$ " similar to $r_{K}$. For the sake of simplicity, $r_{K} "$ may be taken equal to $r_{K}$.

\subsection{Parameters for contractive component of dilatancy}

The fundamental form of the fabric tensor to represent the contractive component of dilatancy in Equation (75) is extended, by using the parameter $r_{\varepsilon_{\mathrm{d}}}$ being common to that for dilative component of dilatancy, and an additional parameter $r_{\varepsilon_{d}^{c}}$ specific to the contractive component of dilatancy, as follows:

$$
\mathbf{I}_{\mathrm{d}}^{\mathrm{c}}=-\frac{1}{4 \pi} r_{\varepsilon_{\mathrm{d}}} r_{\varepsilon_{\mathrm{d}}} r_{S_{0}} \iint r_{\mathrm{t}} \mathrm{M}_{\mathrm{v}}\left(1-c_{1}\left(\frac{G_{\mathrm{L} / \mathrm{U}}}{G_{\mathrm{L} 0}}\right)\right)|\langle\mathbf{t} \otimes \mathbf{n}\rangle|^{*} \mathrm{~d} \omega \mathrm{d} \Omega
$$

This three dimensional form is reduced for two dimensional analysis as

$$
\mathbf{I}_{\mathrm{d}}^{\mathrm{c}}=-r_{\varepsilon_{\mathrm{d}}} r_{\varepsilon_{\mathrm{d}}^{\mathrm{c}}} r_{S_{0}} \int \overline{\mathrm{M}}_{\mathrm{v}}|\langle\mathbf{t} \otimes \mathbf{n}\rangle|^{*} \mathrm{~d} \omega
$$




$$
\overline{\mathrm{M}}_{\mathrm{v}}=r_{\mathrm{t}} \mathrm{M}_{\mathrm{v}}\left(1-c_{1}\left(\frac{G_{\mathrm{L} / \mathrm{U}}}{G_{\mathrm{L} 0}}\right)\right)
$$

The term $r_{S_{0}}$ in Equation (108) controls the rate of increase in magnitude depending on the state of liquefaction and, by using the parameters $q_{1}$ and $q_{2}$, is defined as follows: for $S_{0}^{*}>S_{\mathrm{bi}}(=0.8)$ :

$$
r_{S_{0}}=S_{0}^{*} q_{2}\left[\left(S_{0}^{*}-S_{\mathrm{bi}}\right) q_{1}+\left(1-S_{0}^{*}\right)\right] /\left(1-S_{\mathrm{bi}}\right)
$$

and, for $S_{0}^{*} \leq S_{\mathrm{bi}}(=0.8)$ :

$$
r_{S_{0}}=S_{0}^{*} q_{2}
$$

where

$$
S_{0}^{*}=p^{\prime \prime} / p_{0}
$$

and $S_{0}^{*} \geq S_{1}$.

The parameter $\mathrm{M}_{\mathrm{v}}$ in Equation (109) controlling the contractive component of dilatancy is related with the phase transformation angle $\phi_{\mathrm{p}}$ through

$$
\begin{gathered}
\mathrm{M}_{\mathrm{pv}}=\frac{\mathrm{M}_{\mathrm{p}}}{\sum_{i=1}^{I} \sin \omega_{i} \Delta \omega} \approx \frac{\mathrm{M}_{\mathrm{p}}}{2} \\
\mathrm{M}_{\mathrm{p}}=\sin \phi_{\mathrm{p}}
\end{gathered}
$$

The term in Equation (113) is used, by specifying the upper limit in magnitude for contractive component of dilatancy $\varepsilon_{\mathrm{d}}^{\mathrm{cm}}$ and additional parameter $q_{3}(=1.0)$, to define the term $\mathrm{M}_{\mathrm{v}}$ as follows:

$$
\mathrm{M}_{\mathrm{v}}=\left(1-\frac{\left(-\varepsilon_{\mathrm{d}}^{\mathrm{c}}\right)}{\varepsilon_{\mathrm{d}}^{\mathrm{cm}}}\right)^{q_{3}} \mathrm{M}_{\mathrm{pv}}
$$


where, if $-\varepsilon_{\mathrm{d}}^{\mathrm{c}}>\varepsilon_{\mathrm{d}}^{\mathrm{cm}}$, then $\mathrm{M}_{\mathrm{v}}=0$. For $\varepsilon_{\mathrm{d}}^{\mathrm{cm}}=\infty$, the parameter $\mathrm{M}_{\mathrm{v}}$ is reduced to $\mathrm{M}_{\mathrm{pv}}$.

The term $r_{\mathrm{t}}$ in Equation (109) is introduced, in reference to [36], to impose the limiting line located between the failure and phase transformation lines as follows:

$$
r_{\mathrm{t}}=\left(\mathrm{M}_{\mathrm{tv}}-|q| / p\right) /\left(\mathrm{M}_{\mathrm{tv}}-\mathrm{M}_{\mathrm{r}}\right)
$$

where, if $|q| / p \geq \mathrm{M}_{\mathrm{tv}}$, then $r_{\mathrm{t}}=0$, and, if $|q| / p \leq \mathrm{M}_{\mathrm{r}}$, then $r_{\mathrm{t}}=1$.

In this study, the limit $\mathrm{M}_{\mathrm{tv}}$ is given as an averaged slope of the failure and phase transformation lines in the virtual simple shear mechanism as follows:

$$
\begin{gathered}
\mathrm{M}_{\mathrm{tv}}=\left(\mathrm{M}_{\mathrm{fv}}+\mathrm{M}_{\mathrm{pv}}\right) / 2 \\
\mathrm{M}_{\mathrm{r}}=0.67 \mathrm{M}_{\mathrm{pv}}
\end{gathered}
$$

where $\mathrm{M}_{\mathrm{fv}}$ is given by Equation (88).

\subsection{Parameters for dilative component of dilatancy}

The dilative component of dilatancy for three dimensional analysis in Equation (63) is reduced for two dimensional analysis as

$$
\varepsilon_{\mathrm{d}}^{\mathrm{d}}=r_{\varepsilon_{\mathrm{d}}} \int\left[\left|\frac{\gamma}{\gamma_{\mathrm{v}}}\right|-\ln \left(1+\left|\frac{\gamma}{\gamma_{\mathrm{v}}}\right|\right)\right]_{\mathrm{v}} \mathrm{M}_{\mathrm{fv}} \mathrm{d} \omega
$$

For describing the steady state of soil, Equation (66) is similarly reduced to

$$
\varepsilon_{\mathrm{d}}^{\mathrm{d}}=r_{\varepsilon_{\mathrm{d}}} \int\left[\left|\frac{\tilde{\gamma}}{\gamma_{\mathrm{v}}}\right|-\ln \left(1+\left|\frac{\tilde{\gamma}}{\gamma_{\mathrm{v}}}\right|\right)\right] \gamma_{\mathrm{v}} \mathrm{M}_{\mathrm{fv}} \mathrm{d} \omega
$$

where the term $\left|\tilde{\gamma} / \gamma_{\mathrm{v}}\right|$ is given by Equation (67) using the parameter $\gamma_{\text {vus }}$. This gives the dilative component of dilatancy at the steady state in asymptote as 


$$
\varepsilon_{\mathrm{d} \text { us }}^{\mathrm{d}}=r_{\varepsilon_{d}}\left[\frac{\gamma_{\mathrm{vus}}}{\gamma_{\mathrm{v}}}-\ln \left(1+\frac{\gamma_{\mathrm{vus}}}{\gamma_{\mathrm{v}}}\right)\right] \gamma_{\mathrm{v}} \mathrm{M}_{\mathrm{fv}} \pi
$$

As described earlier, contractive component of dilatancy ceases to increase in its magnitude along the stress path beyond the limiting line specified by Equation (117) and thus keeps the same value assigned at the limiting line when the steady state is eventually reached. This process can be described as follows. First of all, Equation (81) is rewritten in terms of state variable $S$ as

$$
\varepsilon^{\prime}-\varepsilon_{0}{ }^{\prime}=-\frac{1}{1-l_{K}}\left(S^{1-l_{K}}-1\right) \varepsilon_{\mathrm{m} 0}
$$

For a quasi-static undrained shear process keeping the total confining pressure constant, a change in the volume of soil skeleton is the same as the change in the volume of pore water. This process is written, by using porosity $n$, bulk modulus of pore water $K_{\mathrm{f}}$, and initial volumetric strain $\varepsilon_{0}$, as

$$
\varepsilon-\varepsilon_{0}=-\frac{n}{K_{\mathrm{f}}}(1-S) p_{0}
$$

By assuming that the volumetric strain due to dilatancy is zero at the initiation of liquefaction analysis, then $\varepsilon_{0}{ }^{\prime}=\varepsilon_{0}$ to give

$$
\varepsilon-\varepsilon_{0}{ }^{\prime}=\varepsilon-\varepsilon_{0}
$$

Therefore, the dilatancy at the steady state is given, with the state variable being $S=S_{\text {c }}$ and using Equations (122) through (124), by

$$
\varepsilon_{\mathrm{dus}}^{\mathrm{c}}+\varepsilon_{\mathrm{d} \text { us }}^{\mathrm{d}}=\varepsilon_{\mathrm{dus}}=\frac{1}{1-l_{K}}\left(S_{\mathrm{c}}{ }^{1-l_{K}}-1\right) \varepsilon_{\mathrm{m} 0}-\frac{n}{K_{\mathrm{f}}}\left(1-S_{\mathrm{c}}\right) p_{0}
$$

Based on this relationship, if $-\varepsilon_{\mathrm{d}}^{\mathrm{c}}>-\varepsilon_{\mathrm{d} \text { us }}^{\mathrm{c}}\left(=\varepsilon_{\mathrm{d} \text { us }}^{\mathrm{d}}-\varepsilon_{\text {dus }}\right)$, then the dilative component of 
dilatancy at the steady state is updated in association with the current value of $\varepsilon_{\mathrm{d}}^{\mathrm{c}}$ by

$$
\varepsilon_{\mathrm{d} \text { us }}^{\mathrm{d}}=\varepsilon_{\mathrm{dus}}-\varepsilon_{\mathrm{d}}^{\mathrm{c}}
$$

The parameter $\gamma_{\text {vus }}$ is updated also by back-calculation through Equation (121).

The state variable at the steady state $S_{\mathrm{c}}$ is computed from the undrained shear strength $q_{\text {us }}$ as

$$
S_{\mathrm{c}}=q_{\mathrm{us}} / \tau_{\mathrm{m} 0}
$$

where $\tau_{\mathrm{m} 0}=\mathrm{M}_{\mathrm{f}} p_{0}$.

\subsection{Summary of model parameters and examples}

The model parameters described above is summarized in Table I. The strain space multiple mechanism model proposed in this study has 15 primary parameters for the analysis of liquefaction; three specify volumetric mechanism, three specify shear mechanism, and the rest control dilatancy. In particular, the undrained shear strength $q_{\text {us }}$ is treated as one of the input parameters by following the current Japanese practice in design. The undrained shear strength $q_{\text {us }}$ together with other parameters, however, may be tied down with void ratio and other indices that characterizes soils $[41,42]$.

The terms required in incremental form in Equation (45) other than those discussed earlier are derived by simple and straight forward differentiation with respect to the relevant variables. The results are summarized in APPENDIX.

As an example of the model performance, drained behavior during isotropic compression up to the confining pressure of $98 \mathrm{kPa}$ is shown in Figure 9. The parameters shown in Table II are used for the simulation with a power index of $n_{K}=0.5$. By using 
the same parameters except for the parameter $r_{\varepsilon_{d}^{c}}=5.0$, the analysis of a single soil element under defined monotonic simple shear from the various initial confining pressure is shown in Figure 10. Number of the virtual simple multiple mechanisms used for the simulation are 24 for the range from $\omega / 2=0$ to $\pi / 2$. The results shown in Figure 10 indicate that the dilatancy is initially contractive, and then turns into a dilative regime with an increasing shear strain. The tendency to dilate is more significant for a smaller initial confining pressure.

\section{SIMULATION OF STEADY STATE AND CYCLIC MOBILITY}

\subsection{Steady state}

Performance of the strain space multiple mechanism model incorporating the proposed dilatancy model is studied for its capability to simulate steady state and cyclic mobility. Typical steady state behavior of sand identified through the laboratory studies $[42,43]$ is the target of the simulation. In particular, four types of behavior designated by the alphabets A through D in Figure 11 are identified through these laboratory studies. The type A indicates that steady state is reached with monotonic increase in shear stress without decrease in effective confining pressure. The type B indicates that steady state is reached with monotonic increase in shear stress involving decrease in effective confining pressure before going through the phase transformation line. The type $\mathrm{C}$ indicates that steady state is reached after going through a peak in shear stress and quasi-steady state. The type D indicates that steady state is reached after going through a peak.

In Figure 11(b), the slopes (tangential directions) of the stress path curves at the stress 
points on the phase transformation line are in the vertical direction. The slopes (tangential directions) of the stress-strain curves shown in Figure 11(a) coincides with the horizontal direction at quasi-steady state and the stead state. The term 'critical state' widely quoted in the literatures on critical state soil mechanics is not used in this study because this term implies that the steady state are defined on the phase transformation line. In this study, the authors take a generalized view that the steady state may not always be on the phase transformation line.

The standard set of model parameters used for simulation is shown in Table II. Specific parameters varied from the standard set will be described in the relevant parts of the text. The formulation of saturated medium [44] is adopted for the numerical analysis with undrained condition.

The first series of the simulations are performed for undrained condition with monotonic increase in simple shear strain starting from an isotropic stress condition. This loading process is called 'monotonic loading' throughout the paper although the stress might not show a monotonic increase depending on the conditions. The initial confining pressures are varied from 20 to $200 \mathrm{kPa}$. For a small undrained shear strength $q_{\mathrm{us}}=5 \mathrm{kPa}$ with $q_{2}=0.2, r_{\varepsilon_{\mathrm{d}}^{\mathrm{c}}}=30$ and $\varepsilon_{\mathrm{d}}^{\mathrm{cm}}=2.0$, the steady state is reached after going through a peak in shear stress as shown in Figure 12. The results basically represent the type D in Figure 11. However, if the shear strain is kept increasing beyond 20 percent range, shear stress is gradually recovered and reached the specified steady state of $q_{\mathrm{us}}=5 \mathrm{kPa}$ at a large shear strain. The authors are aware of the fact that the small strain/small displacement formulation given in the previous chapters is not applicable to a very large strain range such as shown in Figure 12(a). However, the 
results in this strain range are intentionally shown in order to study the performance of the present formulation as is. Obviously the next step of the study should include the finite strain/large deformation formulation to discuss the applicability of the model to describe actual behavior of soils.

For an intermediate undrained shear strength of $q_{\text {us }}=30 \mathrm{kPa}$ with $r_{\varepsilon_{\mathrm{d}}^{\mathrm{c}}}=30$ shown in Figure 13, the steady state is reached after going through a peak and also going through a quasi-steady state [42], that is more clearly seen than in the case with the small undrained shear strength previously shown in Figure 12. The results basically represent the type $C$ in Figure 11. For an undrained shear strength of $q_{\mathrm{us}}=200 \mathrm{kPa}$ with $r_{\varepsilon_{\mathrm{d}}^{\mathrm{c}}}=30$ shown in Figure 14, similar trend is also observed with a more marked tendency in increase in the shear resistance in the large shear strain range.

In all cases, the steady state specified by the parameter $q_{\text {us }}$ is reached irrespective of the initial confining pressures, being consistent with the results of the studies through laboratory tests $[42,43,45]$.

\subsection{Effects of parameters}

Effects of the parameters $r_{\varepsilon_{\mathrm{d}}^{\mathrm{c}}}, q_{2}$, and $q_{\text {us }}$ are studied more systematically by varying only one parameter at a time and keeping the initial confining pressure the same. The rest of the parameters for dilatancy, i.e. $r_{\varepsilon_{\mathrm{d}}}, q_{1}, S_{1}$, and $c_{1}$, are more or less relevant to the cyclic behavior rather than the steady state; $r_{\varepsilon_{d}}$ controls overall behavior for dilative and contractive component of dilatancy, $q_{1}$ controls initial phase of excess pore water pressure generation, $S_{1}$ has minor effects except for avoiding numerical 
difficulties associated with the condition at zero confining stress, and $c_{1}$ controls threshold limit for liquefaction as described earlier.

The effect of the parameter $r_{\varepsilon_{\mathrm{d}}^{c}}$ ranging from 1 to 50 is shown in Figure 15. Although the same steady state is eventually reached in all cases, transient behavior is significantly affected by this parameter. In particular, for a small $r_{\varepsilon_{\mathrm{d}}^{c}}$, say less than 10 with the given set of the parameters, shear stress monotonically increases with higher rate for smaller $r_{\varepsilon_{\mathrm{d}}^{\mathrm{c}}}$. In particular, the case with $r_{\varepsilon_{\mathrm{d}}^{\mathrm{c}}}=1$ (or less) represents the type A in Figure 11 whereas the cases with $r_{\varepsilon_{\mathrm{d}}^{c}}$ varying from 2 to 10 represent the type B in Figure 11. For a large $r_{\varepsilon_{\mathrm{d}}^{\mathrm{c}}}$, say larger than 20, the shear stress strain path goes through a peak, and then goes through a quasi-steady state before arriving at the steady state, representing the type $\mathrm{C}$ in Figure 11.

The effect of the parameter $q_{2}$ varying from 0 to 5 with $r_{\varepsilon_{d}^{c}}=10$ is more or less restricted to the stress path in the vicinity of phase transformation line as shown in Figure 16. The steady state is reached faster for a larger $q_{2}$.

The effect of $q_{\text {us }}$, if all the rest of the parameters are kept the same with $r_{\varepsilon_{\mathrm{d}}^{\mathrm{c}}}=10$, is not significant for the behavior up to the phase transformation line as shown in Figure 17 (b) and (c), but after that, the effect of $q_{\text {us }}$ becomes significant as shown in Figure 17 (a). The authors are aware of the extreme end of the range set for this parameter as $q_{\mathrm{us}}=1000 \mathrm{kPa}$ that is usually unachievable unless a very high back-pressure is applied but this is intentional to study the model performance as is.

To summarize, the parameter $r_{\varepsilon_{\mathrm{d}}^{\mathrm{c}}}$ controls the strain range in the vicinity of the peak 
as well as around the phase transformation line whereas the parameter $q_{2}$ controls the behavior restricted around the phase transformation line. The parameter $q_{\text {us }}$ primarily governs the behavior at a large strain range. Together with the results shown in Section 6.1, the strain space multiple mechanism model proposed in this study shows a reasonable capability to simulate various types of behavior, including the types A through D shown in Figure 11, being consistent with the laboratory studies $[42,43,45]$

Another interesting feature noted in the stress strain behavior is that, even if the shear stress strain path does not go through a peak, all the stress strain curves show 'a shoulder' located at a relatively small strain range before eventually arriving at the specified steady state as shown in Figure 15 (b) through 17 (b). Since this shoulder might have a significant effect on the deformation of soil, more detailed look into this aspect of soil behavior might be necessary in future.

\subsection{Measured and computed results for monotonic loading}

In order to study the applicability of the strain space multiple mechanism model to typical laboratory results, an attempt is made to simulate a series of the laboratory test results obtained by Yoshimine et al[46]. The laboratory test was performed on Toyoura sand for undrained simple shear condition with the void ratio ranging from $e=0.804$ through 0.888. The parameters used for the simulation varied from those shown in Table II are the internal friction angle $\phi_{\mathrm{f}}=42.7^{\circ}$, with the parameter $q_{2}$ linearly varied from 1.0 to 0.2 , and $r_{\varepsilon_{d}^{c}}$ from 5 to 30. The undrained shear strength was kept at a value large enough (i.e. $500 \mathrm{kPa}$ ) for the simulation with void ratio less than 0.863 . For the void ratio of 0.876 and $0.888, q_{\text {us }}$ of 10 and 1 are used. The measured and computed results 
are shown in Figure 18. The void ratio written for the computed results in Figures 18(b) and (d) are estimated by linear interpolation between $e=0.804$ and 0.888 . Although there are uncertainty in the measured and computed void ratio, the strain space multiple mechanism model demonstrates its capability to capture the essential features of laboratory test results for monotonic loading.

\subsection{Steady state with strain reversal}

The behavior with strain reversal after reaching a steady state is studied with the parameters $q_{\mathrm{us}}=30 \mathrm{kPa}$ and $r_{\varepsilon_{\mathrm{d}}^{\mathrm{c}}}=20$. In this simulation, shear strain is at first increased up to $\gamma_{x y}=40 \%$ towards right in Figure 19 (a) and (b), and then decreased back to zero but kept on going in the negative direction towards left in these figures until another steady state is reached. After this process, the shear strain is reversed again back to zero. This simulation results in an extremely large shear strain in magnitude as shown in Figure 19. It might be too early to make judgment on the validity of the model with respect to this extreme condition of loading because it is difficult to perform laboratory tests with such an extreme loading history. However, the specified undrained shear strength $q_{\text {us }}=30 \mathrm{kPa}$ is confirmed to be reached at both ends of the steady states as the model is intended to.

\subsection{Cyclic behavior}

Performance of the model for cyclic loading under undrained condition is studied without considering the effect of steady state. In this simulation, laboratory test results by Matsuo et al [47] are used as a reference for the simulation. The soil element is at 
first isotropically consolidated with a confining pressure of $p_{0}=65.3 \mathrm{kPa}$, and then is subject to a cyclic simple shear with an amplitude of $\tau_{x y}=14 \mathrm{kPa}$, that corresponds to the stress ratio of $\tau_{x y} / p_{0}=0.21$, for 15 cycles. The parameters varies from those shown in Table II for the simulation includes $\phi_{f}=45^{\circ}, \phi_{p}=26^{\circ}, r_{\varepsilon_{\mathrm{d}}^{c}}=1.2$ and $q_{1}=5$. The measured and computed results are shown in Figure 20. The behavior of sand under this type of loading forms a basis for the studies of one dimensional response and liquefaction of horizontally layered ground during an earthquake. In addition, the relations between the cyclic stress ratio and number of cycles of loading are shown in Figure 21. The results with the double strain amplitudes ranging from 1 to $10 \%$ shown in this figure show the trend similar to the typical laboratory cyclic loading test results such as presented by Inagaki et al [48], basically support the applicability of the strain space multiple mechanism model.

Another type of simulation for cyclic loading is performed in an anisotropic stress field. In this simulation, the soil element is initially consolidated with a vertical confining pressure of $-\sigma_{y 0}{ }^{\prime}=87 \mathrm{kPa}$ with a horizontal confining pressure of $-\sigma_{x 0}{ }^{\prime}=43.5 \mathrm{kPa}$ with an average confining pressure of $p_{0}=65.3 \mathrm{kPa}$. By keeping this axial stress difference constant $\sigma_{x}{ }^{\prime}-\sigma_{y}{ }^{\prime}=43.5 \mathrm{kPa}$ throughout the simulation, a cyclic simple shear with amplitude that corresponds to the same shear stress amplitude of $\tau_{x y}=14 \mathrm{kPa}$ is applied for 10 cycles. The same parameters used for the cyclic loading in the isotropic stress field are used for this simulation. The results are shown in Figure 22. In particular, the stress and strain curve for $\tau_{x y}-\gamma_{x y}$, shown in Figure 22 (a)(b), indicates gradual growth in the amplitude of $\gamma_{x y}$ with overestimation in computed 
results, whereas the axial strain difference $\varepsilon_{x}-\varepsilon_{y}$, shown in Figure 22 (c)(d), exhibits a cumulative increase about the same order in the measured and computed results. These stress strain behaviors are associated with the stress path, shown in Figure 22 (e)(f), that gradually approaches the lower limit in the effective confining pressure. In accordance with this approach, the stress path in the normalized $\tau-p$ plane, shown in Figure 22 (g), approaching a limiting line for contractive component of dilatancy. The behavior of sand under this type of loading forms a basis for the studies on the deformation of soil-structure systems having two dimensional configuration during an earthquake where an anisotropic stress field is imposed due to gravity before the dynamic loading due to the earthquake.

Except for the overestimation in terms of $\gamma_{x y}$ shown in Figure 22, the computed results shown in Figures 20 through 22 reasonably capture the essential features of the sand behavior subject to cyclic loading under undrained condition as studied in the laboratory tests $[49,50]$.

\section{CONCLUSIONS}

A theoretical link between the micromechanical and macroscopic strain energies of granular materials is studied and the results are applied for formulation of dilatancy through a strain space multiple mechanism model. Primary conclusions of this study are summarized as follows:

1) The macroscopic deviator strain energy is given as an integral of microscopic strain 
energy given by the individual virtual simple shear mechanism that constitutes the strain space multiple mechanism model. The macroscopic volumetric strain energy is given as a summation of component due to isotropic stress change, and contractive and dilative components of dilatancy.

2) The classic postulate of interlocking that is defined as the energy less component of deformation forms the basis for dilative component of dilatancy as an integral of those counterparts in the virtual simple shear mechanism. Contractive component of dilatancy is given through an obvious postulate that the macroscopic component is given as an integral of those of microscopic counterparts. The incremental relationship between the macroscopic volumetric strain due to dilatancy and the macroscopic strain tensor is formulated through second order fabric tensors.

3) Performance of the dilatancy model described above is demonstrated for monotonic and cyclic loading under undrained condition. In particular, the model shows reasonable capability to simulate the essential features of the behavior of sand, including the peak, quasi-steady state, and steady state for monotonic loading. The model also shows reasonable performance to simulate cyclic behavior of sand under isotropic and anisotropic stress fields.

\section{APPENDIX}

Three dimensional multiple mechanism model generalized in Chapter 3 is reduced to two dimensional multiple mechanism model as follows. In two dimension, Equations (33) and(35) are reduced to

$$
q=q\left(\gamma, \varepsilon^{\prime}, \varepsilon^{\prime \prime}\right)
$$




$$
\dot{\varepsilon}_{\mathrm{d}}=\mathbf{I}_{\mathrm{d}}: \dot{\boldsymbol{\varepsilon}}
$$

Consequently the generalized incremental constitutive equation in Equation (44) is given by

$$
\dot{\boldsymbol{\sigma}}^{\prime}=\square: \dot{\boldsymbol{\varepsilon}}
$$

$$
\begin{aligned}
\square= & K_{\mathrm{L} / \mathrm{I}} \mathbf{I} \otimes \mathbf{I}+\int G_{\mathrm{L} / \mathrm{U}}\langle\mathbf{t} \otimes \mathbf{n}\rangle \otimes\langle\mathbf{t} \otimes \mathbf{n}\rangle \mathrm{d} \omega-K_{\mathrm{L} / \mathrm{U}} \mathbf{I} \otimes \mathbf{I}_{\mathrm{d}} \\
& +\int H\langle\mathbf{t} \otimes \mathbf{n}\rangle \mathrm{d} \omega \otimes\left(\mathbf{I}-\mathbf{I}_{\mathrm{d}}\right)+\int L\langle\mathbf{t} \otimes \mathbf{n}\rangle \mathrm{d} \omega \otimes\left(\mathbf{I}-\mathbf{I}_{\mathrm{d}}^{\mathrm{c}}\right)
\end{aligned}
$$

Differentiation with respect to the relevant variables yields the terms $K_{\mathrm{L} / \mathrm{U}}$, $G_{\mathrm{L} / \mathrm{U}}, H, L, \mathbf{I}_{\mathrm{d}}$ and $\mathbf{I}_{\mathrm{d}}^{\mathrm{c}}$ in Equation (131). Among these terms, the terms $K_{\mathrm{L} / \mathrm{U}}, G_{\mathrm{L} / \mathrm{U}}$, and $\mathbf{I}_{\mathrm{d}}^{\mathrm{c}}$ are given in Equations (78) through (80), (89), and (108) in the main text. After some straight forward manipulation, the terms $H$ and $L$ are given as follows:

$$
H=\frac{\partial q}{\partial \varepsilon^{\prime}}=-K_{\mathrm{L} / \mathrm{U}}\left(\frac{\partial q}{\partial q_{\mathrm{v}}} \frac{\partial q_{\mathrm{v}}}{\partial \tau_{\mathrm{m}}} \frac{\partial \tau_{\mathrm{m}}}{\partial p}+\frac{\partial q}{\partial \gamma_{\mathrm{v}}} \frac{\partial \gamma_{\mathrm{v}}}{\partial \gamma_{\mathrm{m}}} \frac{\partial \gamma_{\mathrm{m}}}{\partial p}\right)
$$

and, if $S_{0}<S_{0 \mathrm{bd}}, \mathrm{d} p "<0$ and $S_{0} \geq p " / p_{0}$, then

$$
L=\frac{\gamma_{\mathrm{m}}}{S_{0}} \frac{\partial q}{\partial \gamma_{\mathrm{v}}} \frac{\partial \gamma_{\mathrm{v}}}{\partial \gamma_{\mathrm{m}}} \frac{K_{\mathrm{L} / \mathrm{U}} "}{p_{0}}
$$

otherwise $L=0$. The terms in Equations (132) and (133) are given, for the backbone curve, by

$$
\begin{gathered}
\frac{\partial q}{\partial q_{\mathrm{v}}}=\frac{\gamma / \gamma_{\mathrm{v}}}{1+\left|\gamma / \gamma_{\mathrm{v}}\right|} \\
\frac{\partial q}{\partial \gamma_{\mathrm{v}}}=-\frac{\gamma / \gamma_{\mathrm{v}}}{\left(1+\left|\gamma / \gamma_{\mathrm{v}}\right|\right)^{2}} \frac{q_{\mathrm{v}}}{\gamma_{\mathrm{v}}} \\
\frac{\partial \tau_{m}}{\partial p}=\mathrm{M}_{\mathrm{f}}
\end{gathered}
$$




$$
\frac{\partial \gamma_{\mathrm{m}}}{\partial p}=\left\{\begin{array}{cc}
0 & \text { (for liquefaction analysis) } \\
\left(1-m_{G}\right) \mathrm{M}_{\mathrm{f}} / G_{\mathrm{m}} & \text { (for static gravity analysis) }
\end{array}\right.
$$

and the rest of the terms are given by Equations (86) and (87). For the formulation of hysteretic part, see the reference [38].

The second order fabric tensor for dilative component of dilatancy for liquefaction analysis $\mathbf{I}_{\mathrm{d}}^{\mathrm{d}}$ is given, if $S_{0}<S_{0 \mathrm{bd}}, \mathrm{d} p "<0$ and $S_{0} \geq p^{\prime \prime} / p_{0}$, by

$$
\mathbf{I}_{\mathrm{d}}^{\mathrm{d}}=r_{\varepsilon_{\mathrm{d}}} \int \frac{\gamma / \gamma_{\mathrm{v}}}{1+\left|\gamma / \gamma_{\mathrm{v}}\right|} \mathrm{M}_{\mathrm{fv}}\langle\mathbf{t} \otimes \mathbf{n}\rangle \mathrm{d} \omega+\frac{\partial \varepsilon_{\mathrm{d}}^{\mathrm{d}}}{\partial \gamma_{\mathrm{v}}} \frac{\gamma_{\mathrm{v}}}{S_{0}} \frac{K_{\mathrm{L} / \mathrm{U}} "}{p_{0}}\left(\mathbf{I}-\mathbf{I}_{\mathrm{d}}^{\mathrm{c}}\right)
$$

and, otherwise, by

$$
\mathbf{I}_{\mathrm{d}}^{\mathrm{d}}=r_{\varepsilon_{\mathrm{d}}} \int \frac{\gamma / \gamma_{\mathrm{v}}}{1+\left|\gamma / \gamma_{\mathrm{v}}\right|} \mathrm{M}_{\mathrm{fv}}\langle\mathbf{t} \otimes \mathbf{n}\rangle \mathrm{d} \omega
$$

where

$$
\frac{\partial \varepsilon_{\mathrm{d}}^{\mathrm{d}}}{\partial \gamma_{\mathrm{v}}}=r_{\varepsilon_{d}} \int\left(\frac{\left|\gamma / \gamma_{\mathrm{v}}\right|}{1+\left|\gamma / \gamma_{\mathrm{v}}\right|}-\ln \left(1+\left|\frac{\gamma}{\gamma_{\mathrm{v}}}\right|\right)\right) \mathrm{M}_{\mathrm{fv}} \mathrm{d} \omega
$$

Finally the second order fabric tensor for dilatancy is given by

$$
\mathbf{I}_{\mathrm{d}}=\mathbf{I}_{\mathrm{d}}^{\mathrm{c}}+\mathbf{I}_{\mathrm{d}}^{\mathrm{d}}
$$

For representing steady state, the second order fabric tensor $\mathbf{I}_{\mathrm{d}}^{\mathrm{d}}$ is given, if $S_{0}<S_{0 \mathrm{bd}}$,

$$
\begin{aligned}
& \mathrm{d} p "<0 \text { and } S_{0} \geq p " / p_{0} \text {, by } \\
& \qquad \mathbf{I}_{\mathrm{d}}^{\mathrm{d}}=r_{\varepsilon_{\mathrm{d}}} \int \frac{\tilde{\gamma} / \gamma_{\mathrm{v}}}{1+\left|\tilde{\gamma} / \gamma_{\mathrm{v}}\right|} \operatorname{sgn}(\gamma) \exp \left(-\frac{|\gamma|}{\gamma_{\text {vus }}}\right) \mathrm{M}_{\mathrm{fv}}\langle\mathbf{t} \otimes \mathbf{n}\rangle \mathrm{d} \omega+\frac{\partial \varepsilon_{\mathrm{d}}^{\mathrm{d}}}{\partial \gamma_{\mathrm{v}}} \frac{\gamma_{\mathrm{v}}}{S_{0}} \frac{K_{\mathrm{L} / \mathrm{U}}}{p_{0}}\left(\mathbf{I}-\mathbf{I}_{\mathrm{d}}^{\mathrm{c}}\right)
\end{aligned}
$$

otherwise

$$
\mathbf{I}_{\mathrm{d}}^{\mathrm{d}}=r_{\varepsilon_{\mathrm{d}}} \int \frac{\tilde{\gamma} / \gamma_{\mathrm{v}}}{1+\left|\tilde{\gamma} / \gamma_{\mathrm{v}}\right|} \operatorname{sgn}(\gamma) \exp \left(-\frac{|\gamma|}{\gamma_{\text {vus }}}\right) \mathbf{M}_{\mathrm{fv}}\langle\mathbf{t} \otimes \mathbf{n}\rangle \mathrm{d} \omega
$$

where 


$$
\frac{\partial \varepsilon_{\mathrm{d}}^{\mathrm{d}}}{\partial \gamma_{\mathrm{v}}}=r_{\varepsilon_{d}} \int\left(\frac{\left|\tilde{\gamma} / \gamma_{\mathrm{v}}\right|}{1+\left|\gamma / \gamma_{\mathrm{v}}\right|}-\ln \left(1+\left|\frac{\tilde{\gamma}}{\gamma_{\mathrm{v}}}\right|\right)\right) \mathrm{M}_{\mathrm{fv}} \mathrm{d} \omega
$$

and the term $\left|\tilde{\gamma} / \gamma_{\mathrm{v}}\right|$ is given by Equation (67) using the parameter $\gamma_{\text {vus }}$.

For static gravity analysis, the second order fabric tensor for dilatancy $\mathbf{I}_{\mathrm{d}}$ is given by

$\mathbf{I}_{\mathrm{d}}=\left(1-K_{\mathrm{L} / \mathrm{U}} \frac{\partial \varepsilon_{\mathrm{d}}^{\mathrm{d}}}{\partial \gamma_{\mathrm{v}}} \frac{\partial \gamma_{\mathrm{v}}}{\partial \gamma_{\mathrm{m}}} \frac{\partial \gamma_{\mathrm{m}}}{\partial p}\right)^{-1}\left[\mathbf{I}_{\mathrm{d}}^{\mathrm{c}}+\left[r_{\varepsilon_{\mathrm{d}}} \int \frac{\gamma / \gamma_{\mathrm{v}}}{1+\left|\gamma / \gamma_{\mathrm{v}}\right|} \mathbf{M}_{\mathrm{fv}}\langle\mathbf{t} \otimes \mathbf{n}\rangle \mathrm{d} \omega-K_{\mathrm{L} / \mathrm{U}} \frac{\partial \varepsilon_{\mathrm{d}}^{\mathrm{d}}}{\partial \gamma_{\mathrm{v}}} \frac{\partial \gamma_{\mathrm{v}}}{\partial \gamma_{\mathrm{m}}} \frac{\partial \gamma_{\mathrm{m}}}{\partial p} \mathbf{I} \mid\right]_{]}\right]$

\section{REFERENCES}

1. Towhata I, Ishihara K. Modelling soil behaviour under principal stress axes rotation. In Proc 5th Int Conf on Numerical Methods in Geomechanics. Balkema: Nagoya, $1985 ; 523-530$.

2. Iai S, Matsunaga Y, Kameoka T. Strain space plasticity model for cyclic mobility. Soils and Foundations 1992; 32(2):1-15.

3. Iai S, Matsunaga Y, Kameoka T. Analysis of undrained cyclic behavior of sand under anisotropic consolidation. Soils and Foundations 1992; 32(2):16-20.

4. Iai S, Kameoka T, Towhata I. Analysis of non-coaxiality by multi-mechanism model. In Proc 8th International Conference on Computer Methods and Advances in Geomechanics: Morgantown, 1994; 599-604.

5. Iai S, Kameoka T, Matsunaga Y. Numerical (Class A) prediction. In Verification of Numerical Procedures for the Analysis of Soil Liquefaction Problems. Balkema, 1993; 109-127, 369-375, 939-946, 1035-1040.

6. Iai S, Morita T, Kameoka T, Matsunaga Y, Abiko K. Response of a dense sand deposit during 1993 Kushiro-Oki earthquake. Soils and Foundations 1995; 35(1):115-131.

7. Iai S, Kameoka T. Finite element analysis of earthquake induced damage to anchored sheetpile quay walls. Soils and Foundations 1993; 33(1):71-91.

8. Iai S. Seismic analysis and performance of retaining structures. Geotechnical Earthquake Engineering and Soil Dynamics III: Geotechnical Special Publication No.75, ASCE; $1998 . \quad$ 1020-1044.

9. Iai S, Ichii K, Sato Y, Kuwajima R. Earthquake response analysis of a high embankment on an existing hill slope. In Proc 2nd International Conference on Earthquake Geotechnical Engineering. Balkema: Lisbon, 1999; 697-702.

10. Ozutsumi O, Sawada S, Iai S, Takeshima Y, Sugiyama W, Shimazu T. Effective stress analyses of liquefaction-induced deformation in river dikes. Soil Dynamics and Earthquake Engineering 2002; 22::1075-1082.

11. Iai S, Ichii K, Liu H, Morita T. Effective stress analyses of port structures. Soils and Foundations, Special Issue on Geotechnical Aspects of the January 171995 Hyogoken-Nambu Earthquake 1998; 2::97-114.

12. Iai S, Matsunaga Y. Mechanism of uplift of underground structures due to liquefaction. In Proc International Symposium on Natural Disaster Reduction and Civil Engineering. JSCE: Osaka, 1991; 297-306. 
13. Towhata I, Ishihara K. Shear work and pore water pressure in undrained shear. Soils and Foundations 1985; 25(3):73-85.

14. Fang HL. A state-dependent multi-mechanism model for sands. Geotechnique 2003; 53(4):407-420.

15. Nishimura S, Towhata I. Three dimensional stress strain behavior of sand during rotation of principal stress axes. Soils and Foundations 2004; 44(2):103-116.

16. Thornton C. A direct approach to micromechanically based continuum models for granular material. In Mechanics of Granular Materials JSSMFE: XII ICSMFE, $1989 ; 145-150$.

17. Mehrabadi MM, Nemat-Nasser S, Oda M. On statistical description of stress and fabric in granular materials. International Journal for Numerical and Analytical Methods in Geomechanics 1982; 6(1):95-108.

18. Iai S. Micromechanical background to a strain space multiple mechanism model for sand. Soils and Foundations 1993; 33(1):102-117.

19. Iai S, Ozutsumi O. Yield and cyclic behaviour of a strain space multiple mechanism model for granular materials. International Journal for Numerical and Analytical Methods in Geomechanics 2005; 29(4):417-442.

20. Iai S. Three dimensional formulation and objectivity of a strain space multiple mechanism model for sand. Soils and Foundations 1993; 33(1):102-117.

21. Akiyoshi T, Matsumoto H, Fuchida K, Fang HL. Cyclic mobility behaviour of sand by the three-dimensional strain space multimechanism model. International Journal for Numerical and Analytical Methods in Geomechanics 1994; 18(6):397-415.

22. Oda M, Nemat-Nasser S, Mehrabadi M. A statistical study of fabric in a random assembly of spherical granules. International Journal for Numerical and Analytical Methods in Geomechanics 1982; 6(1):77-94.

23. Iai S. Concept of effective strain in constitutive modeling of granular materials. Soils and Foundations 1993; 33(2):171-180.

24. Hardin BO, Drnevich VP. Shear modulus and damping in soils: design equation and curves. Journal of Soil Mechanics and Foundations Division, ASCE 1972; 98(SM7):667-692.

25. Batdorf SB, Budiansky B. A mathematical theory of plasticity based on the concept of slip. National Advisory Committee for Aeronautics 1949:TN 1871.

26. Calladine CR. A microstructural view of the mechanical properties of saturated clay. Geotechnique 1971; 21(4):391-415.

27. Pande GN, Sharma KG. Mutilaminate model of clays - a numerical evaluation of the influence of rotation of principal stress axes. International Journal for Numerical and Analytical Methods in Geomechanics 1983; 7(4):397-418.

28. Yin ZY, Chang CS. Non-uniqueness of critical state line in compression and extension conditions. International Journal for Numerical and Analytical Methods in Geomechanics 2009; 33(10):1315-1338.

29. Fukutake K, Matsuoka H. A unified law for dilatancy under multil-directional simple shearing. Proc of JSCE 1989; 412(III-12):143-151(in Japanese).

30. Iai S, Matsunaga Y, Kameoka T. Parameter identification for a cyclic mobility model. Report of the Port and Harbour Research Institute 1990; 29(4):57-83.

31. Ozutsumi O, Iai S. Adjustment method of the hysteresis damping for multiple shear spring model. In Proc 4th International Conference on Recent Advances in Geotechnical Earthquake Engineering and Soil Dynamics, 2001; Paper No.1.68.

32. Taylor DW. Fundamentals of Soil Mechanics: John Wiley \& Sons; 1948. 700 p.

33. Roscoe KH, Schofield AN, Thurairajah A. Yielding of clays in states wetter than critical. Geotechnique 1963; 13(3):211-240.

34. Schofield AN, Wroth CP. Critical State Soil Mechanics. London: McGraw-Hill; 1968.

35. Iai S. A new look at the stress dilatancy relation in Cam-Clay model. Soils and Foundations 1994; 34(2):1-12.

36. Ozutsumi O, Shiozaki Y, Ichii K, Iai S, Mori G. Study of the improvement of accuracy 
for the 2-dimensional effective stress analysis method. Annual Journal of Civil Engineering in the Ocean, JSCE 2004; 20::443-448 (in Japanese).

37. Collins IF. The concept of stored plastic work or frozen elastic energy in soil mechanics. Geotechnique 2005; 55(5):373-382.

38. Iai S, Tobita T, Ozutsumi O. Stress dilatancy relation in strain space multiple mechanism model for cyclic behavior of sand. Annuals of Disaster Prevention Research Institute, Kyoto University 2008; 51::291-304 (in Japanese).

39. Ishihara K, Yoshimine M. Evaluation of settlements in sand deposits following liquefaction during earthquakes. Soils and Foundations 1992; 32(1):173-188.

40. Koseki J, Kawakami S, Nagayama H, Sato T. Change of small strain quasi-elastic deformation properties during undrained cyclic torsional shear and triaxial tests of Toyoura sand. Soils and Foundations 2000; 40(3):101-110.

41. Been K, Jefferies MG. A state parameter for sands. Geotechnique 1985; 35(2):99-112.

42. Ishihara K. Liquefaction and flow failure during earthquakes. Geotechnique 1993; 43(3):351-415.

43. Yoshimine M, Ishihara K. Flow potential of sand during liquefaction. Soils and Foundations 1998; 38(3):189-198.

44. Zienkiewicz OC, Bettess P. Soils and other saturated media under transient, dynamic conditions. In: Pande, Zienkiewicz, editors. Soil Mechanics - Transient and Cyclic Loads: John Wiley and Sons; 1982. 1-16.

45. Verdugo R. Characterization of sandy soil behavior under large deformation: The University of Tokyo. 1992.

46. Yoshimine M, Ishihara K, Vargas W. Effects of principal stress direction and intermediate principal stress on undrained shear behavior of sand. Soils and Foundations 1998; 38(3):179-188.

47. Matsuo O, Shimazu T, Uzuoka R, Mihara M, Nishi K. Numrical analysis of seismic behavior of embankments founded on liquefiable soils. Soils and Foundations 2000; 40(2):21-39.

48. Inagaki H, Iai S, Sugano T, Yamazaki H, Inatomi T. Performance of caisson type quay walls at Kobe port. Soils and Foundations, Special Issue on Geotechnical Aspects of the January 171995 Hyogoken-Nambu Earthquake 1996:119-136.

49. Ishihara K. Stability of natural deposits during earthquakes. In Proceedings of 11th International Conference on Soil Mechanics and Foundation Engineering. Balkema: San Francisco, 1985; 327-376.

50. Ishihara K, Li S. Liquefaction of saturated sand in triaxial torsion shear test. Soils and Foundations 1972; 12(2):19-39. 
Table I. Model parameters.

\begin{tabular}{lll}
\hline Symbol & Mechanism & Parameter designation \\
\hline$K_{\mathrm{L} / \mathrm{Ua}}$ & Volumetric & Bulk modulus \\
$r_{K}$ & Volumetric & Reduction factor of bulk modulus for liquefaction analysis \\
$l_{K}$ & Volumetric & Power index of bulk modulus for liquefaction analysis \\
$G_{\mathrm{ma}}$ & Shear & Shear modulus \\
$\phi_{\mathrm{f}}$ & Shear & Internal friction angle \\
$h_{\text {max }}$ & Shear & Upper bound for hysteretic damping factor \\
$\phi_{\mathrm{p}}$ & Dilatancy & Phase transformation angle \\
$r_{\varepsilon_{\mathrm{d}}}$ & Dilatancy & Parameter controlling dilative and contractive components \\
$r_{\varepsilon_{\mathrm{d}}^{\mathrm{c}}}$ & Dilatancy & Parameter controlling contractive component \\
$q_{1}$ & Dilatancy & Parameter controlling initial phase of contractive component \\
$q_{2}$ & Dilatancy & Parameter controlling final phase of contractive component \\
$\varepsilon_{\mathrm{d}}^{\mathrm{cm}}$ & Dilatancy & Limit of contractive component \\
$S_{1}$ & Dilatancy & Small positive number to avoid zero confining pressure \\
$c_{1}$ & Dilatancy & Parameter controlling elastic range for contractive component \\
$q_{\mathrm{us}}$ & Dilatancy & Undrained shear strength (for steady state analysis) \\
\hline
\end{tabular}

Table II. Model parameters used as the standard set for simulation.

\begin{tabular}{|c|c|c|c|c|c|c|c|c|c|c|c|c|c|c|}
\hline $\begin{array}{l}K_{\mathrm{L} / \mathrm{Ua}} \\
(\mathrm{kPa})\end{array}$ & $r_{K}$ & $l_{K}$ & $\begin{array}{l}G_{\text {ma }} \\
(\mathrm{kPa})\end{array}$ & $\begin{array}{l}\phi_{\mathrm{f}} \\
\left(^{\circ}\right)\end{array}$ & $h_{\max }$ & $\begin{array}{l}\phi_{\mathrm{p}} \\
\left(^{\circ}\right)\end{array}$ & $r_{\varepsilon_{\mathrm{d}}}$ & $r_{\varepsilon_{\mathrm{d}}^{\mathrm{c}}}$ & $q_{1}$ & $q_{2}$ & $\varepsilon_{\mathrm{d}}^{\mathrm{cm}}$ & $S_{1}$ & $C_{1}$ & $\begin{array}{l}q_{\text {us }} \\
(\mathrm{kPa})\end{array}$ \\
\hline 220300 & 0.5 & 2 & 84490 & 39.7 & 0.24 & 28.0 & 0.1 & 1.5 & 1.0 & 1.0 & 0.2 & 0.005 & 1.0 & 200 \\
\hline
\end{tabular}




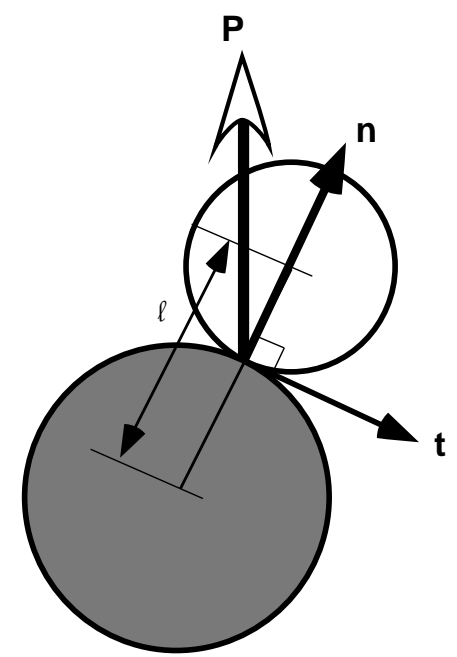

Figure 1. Contact normal $\mathbf{n}$, tangential direction $\mathbf{t}$ and contact force $\mathbf{P}$.

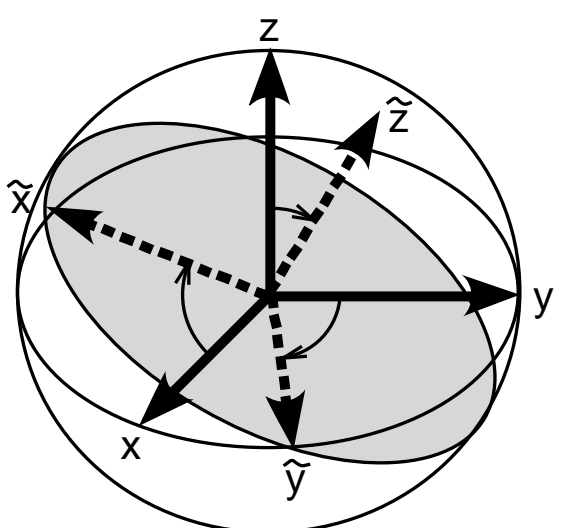

Figure 2. Local co-ordinate $\tilde{\mathbf{x}}, \tilde{\mathbf{y}}, \tilde{\mathbf{z}}$ for defining the virtual two dimensional mechanism.

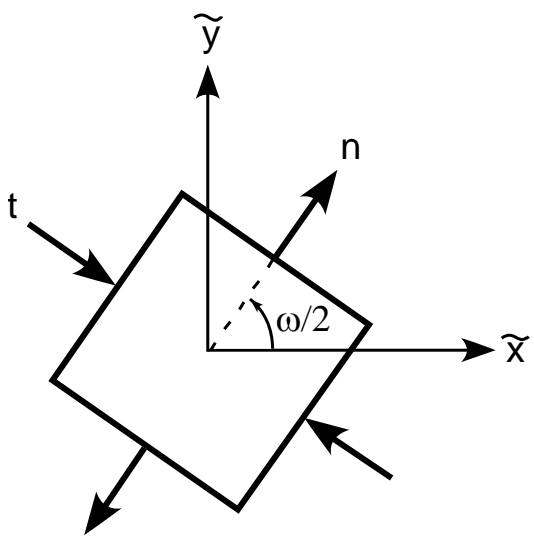

(a)

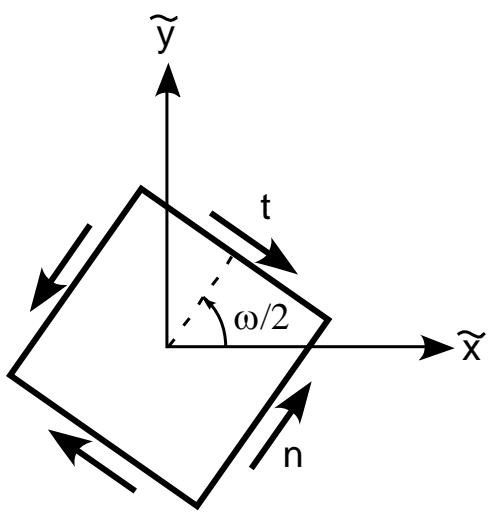

(b)

Figure 3. Stress component in the direction of $\omega / 2$ relative to the reference local coordinate $\tilde{\mathbf{x}}$ in the virtual plane for defining two dimensional mechanisms: (a) biaxial shear $\langle\mathbf{n} \otimes \mathbf{n}\rangle$; (b) simple shear $\langle\mathbf{t} \otimes \mathbf{n}\rangle$. 


\section{MACROSCOPIC STRAIN FIELD}

projection

micromechanical (virtual) strain

non-linear transformation

micromechanical (virtual) stress

tensorial average

MACROSCOPIC STRESS FIELD

\section{MACROSCOPIC STRESS FIELD}

projection

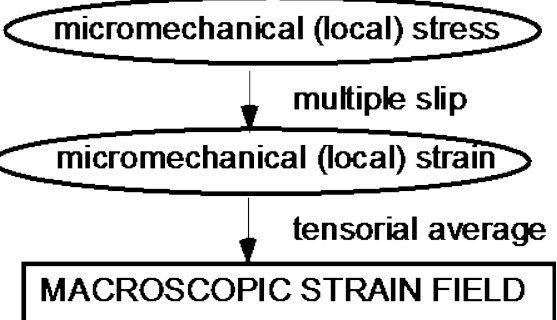

Figure 4. Conceptual background to the two families of multiple mechanism models.
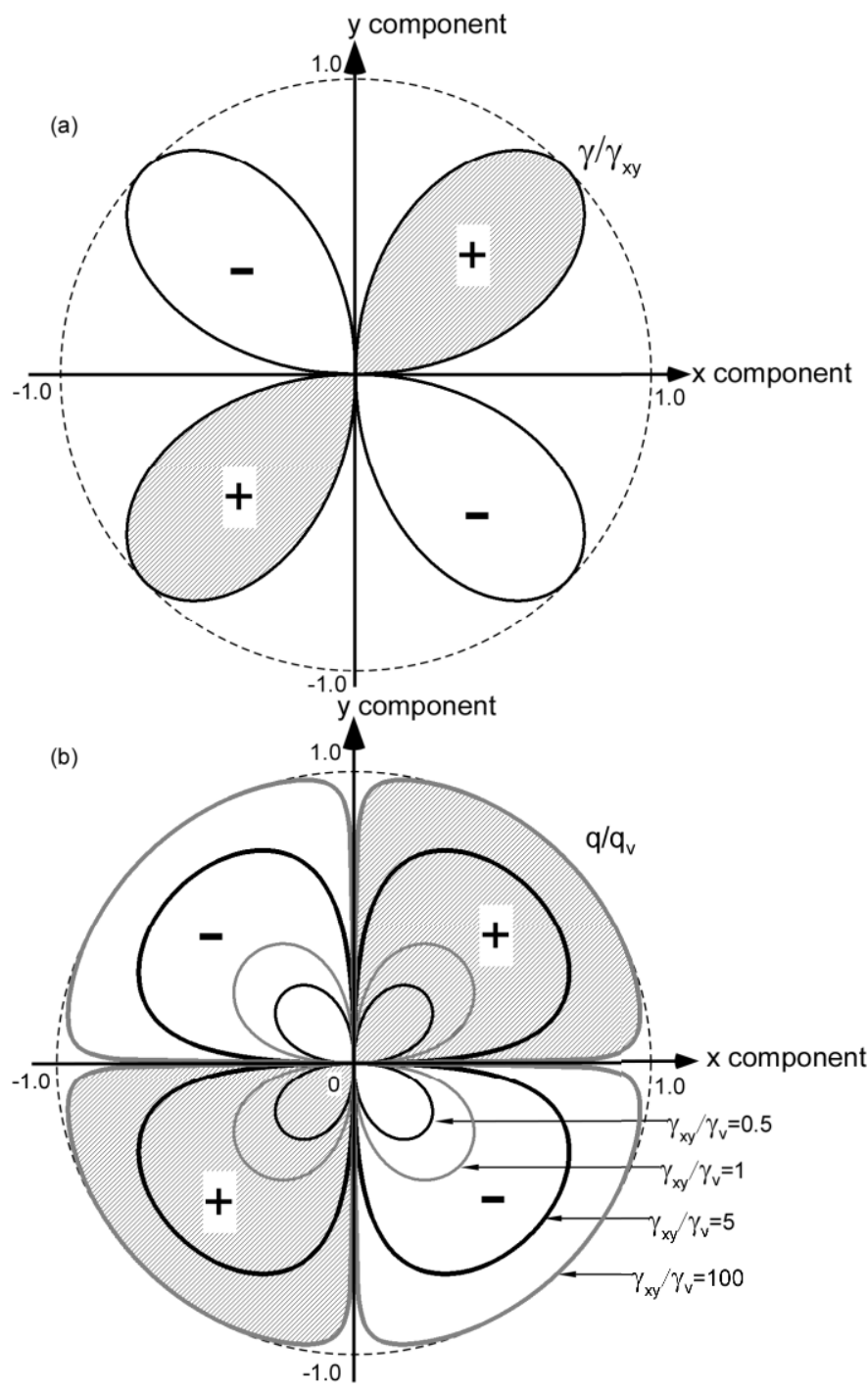

Figure 5. Evolution of fabric mobilized by macroscopic simple shear in $\boldsymbol{x}$-y plane: (a) fabric in strain space; (b) fabric in stress space. 


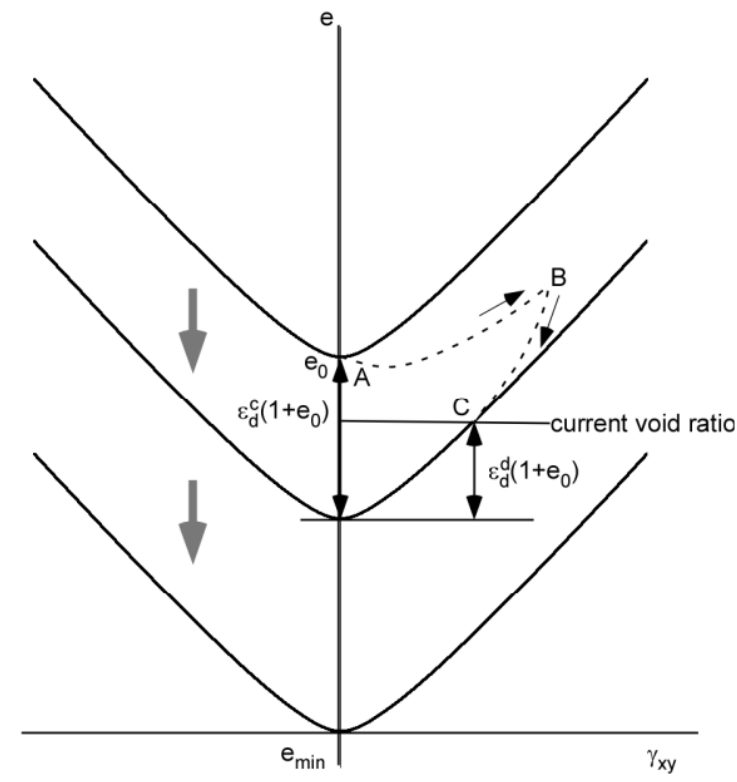

Figure 6. Schematic figure for dilative and contractive components of dilatancy.

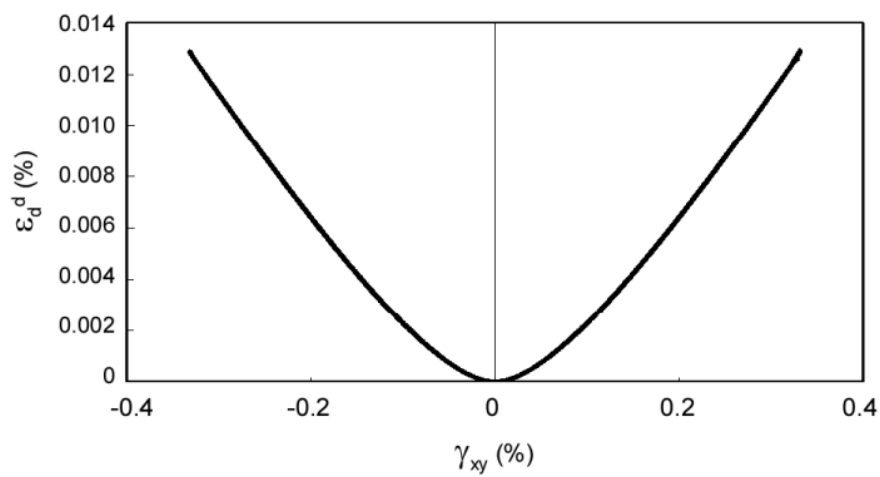

Figure 7. Dilative component of dilatancy $\left(r_{\varepsilon_{\mathrm{d}}}=0.1, \mathbf{M}_{\mathrm{fv}}=0.32\right.$ and $\left.\gamma_{\mathrm{v}}=5.8 \times 10^{-4}\right)$.

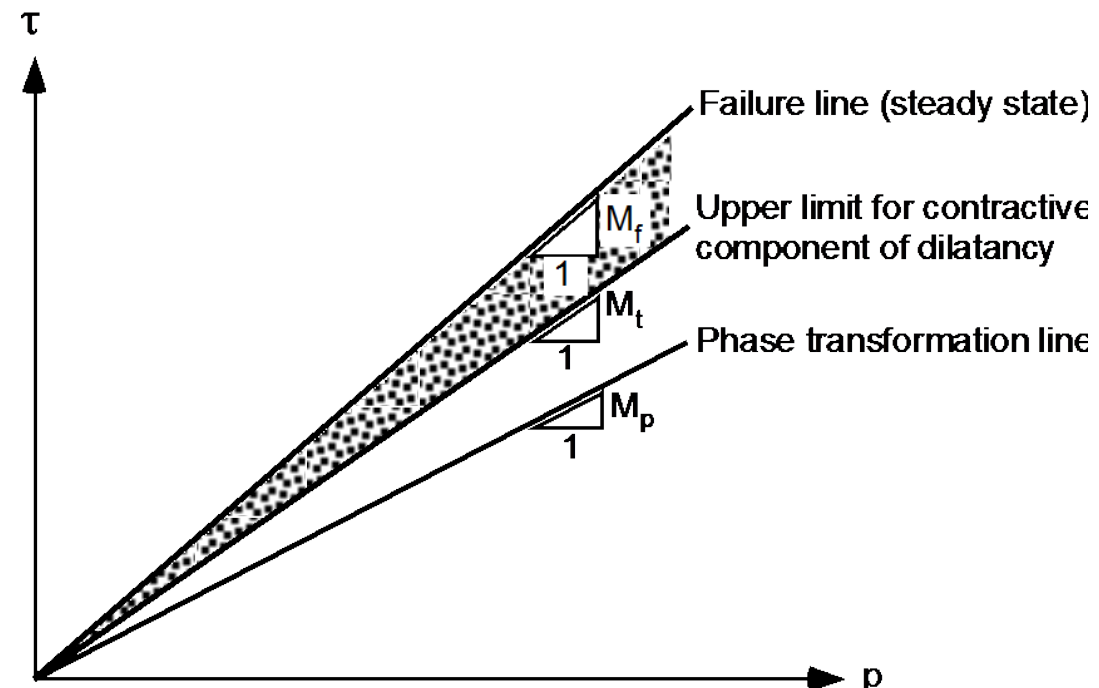

Figure 8. Zoning of stress plane with respect to dilatancy. 


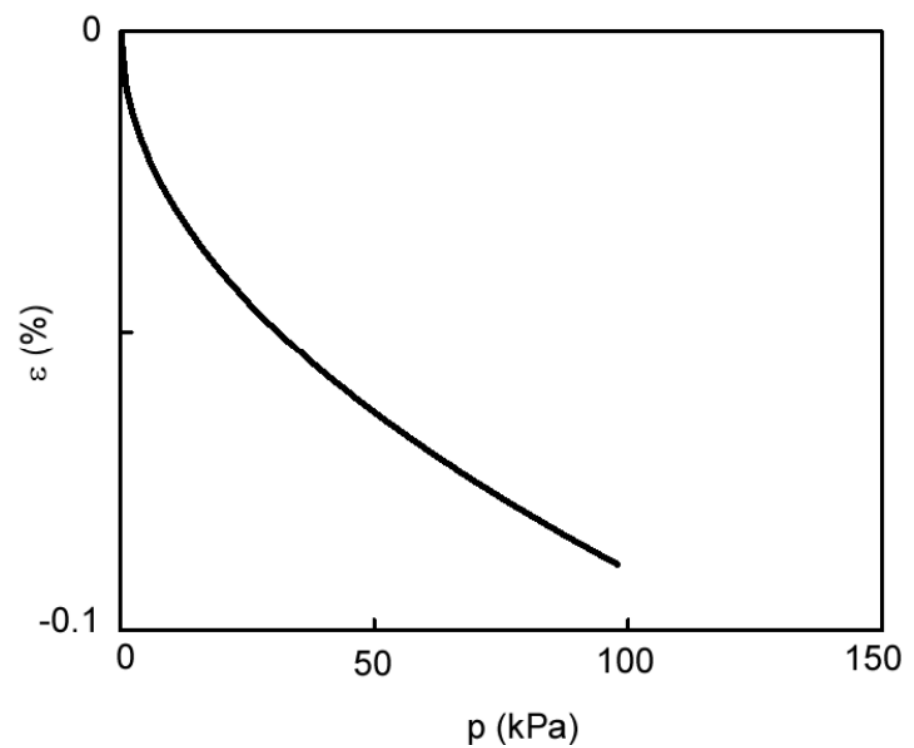

Figure 9. Drained behavior during isotropic compression.
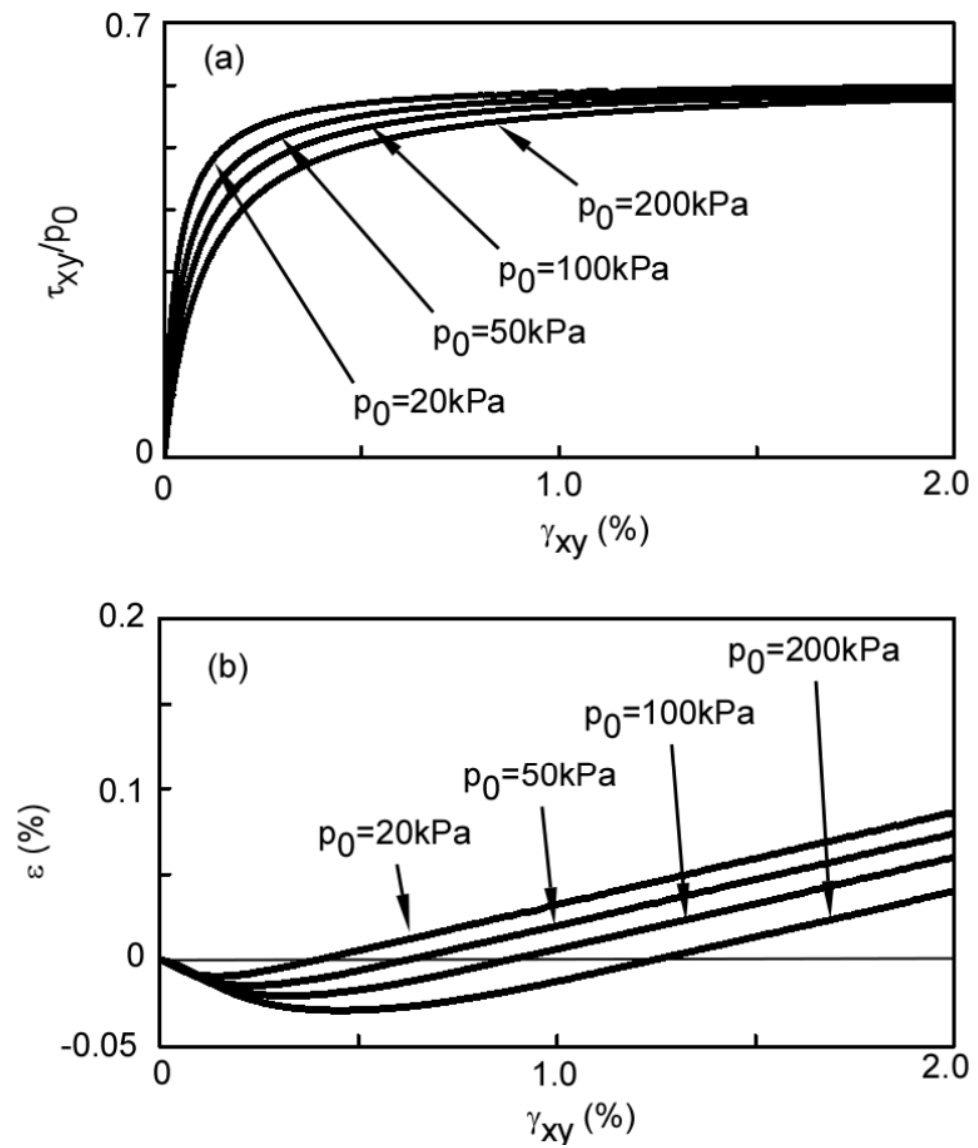

Figure 10. Drained behavior under monotonic loading; (a) stress-strain relationship; (b) volumetric strain-shear strain relationship. 


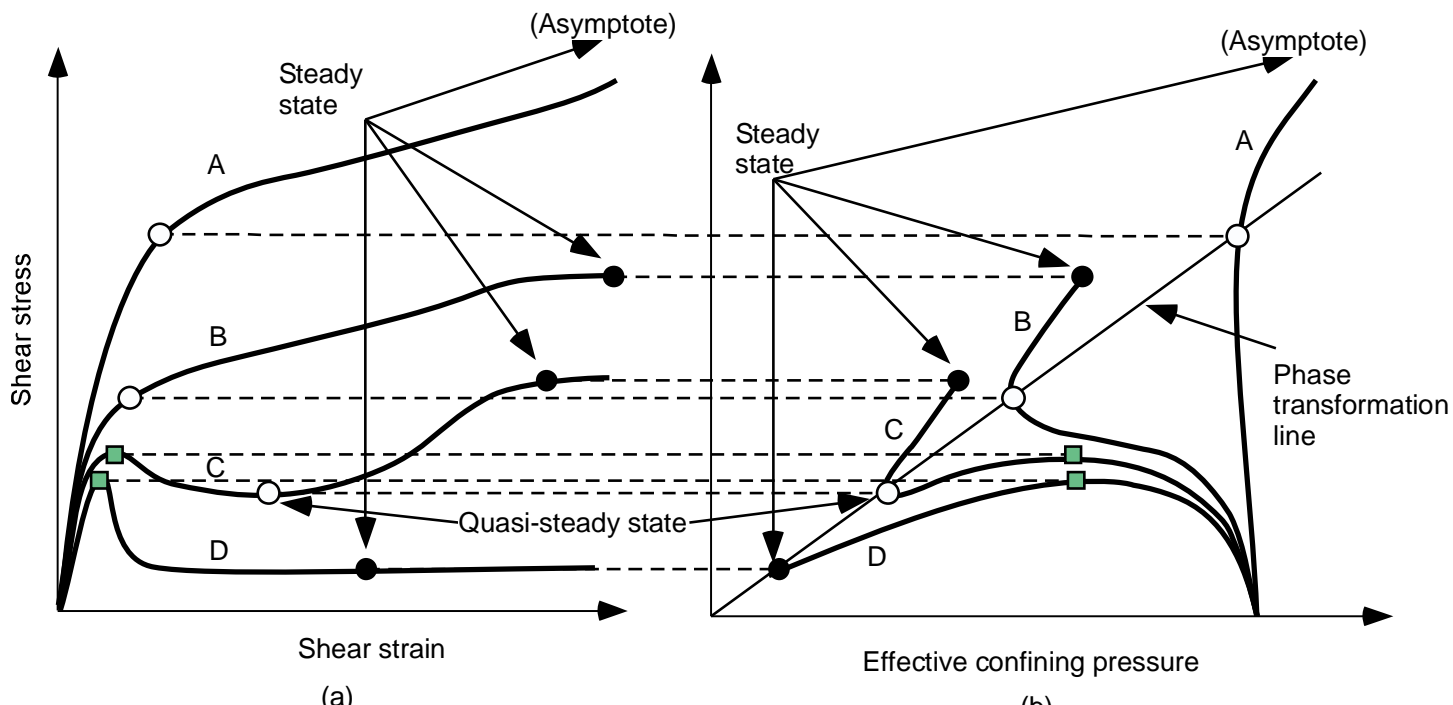

(a)

(b)

Figure 11. Schematic figure of undrained behavior of sand under monotonic loading (modified after [43]): (a) stress-strain relationship; (b) stress path. 

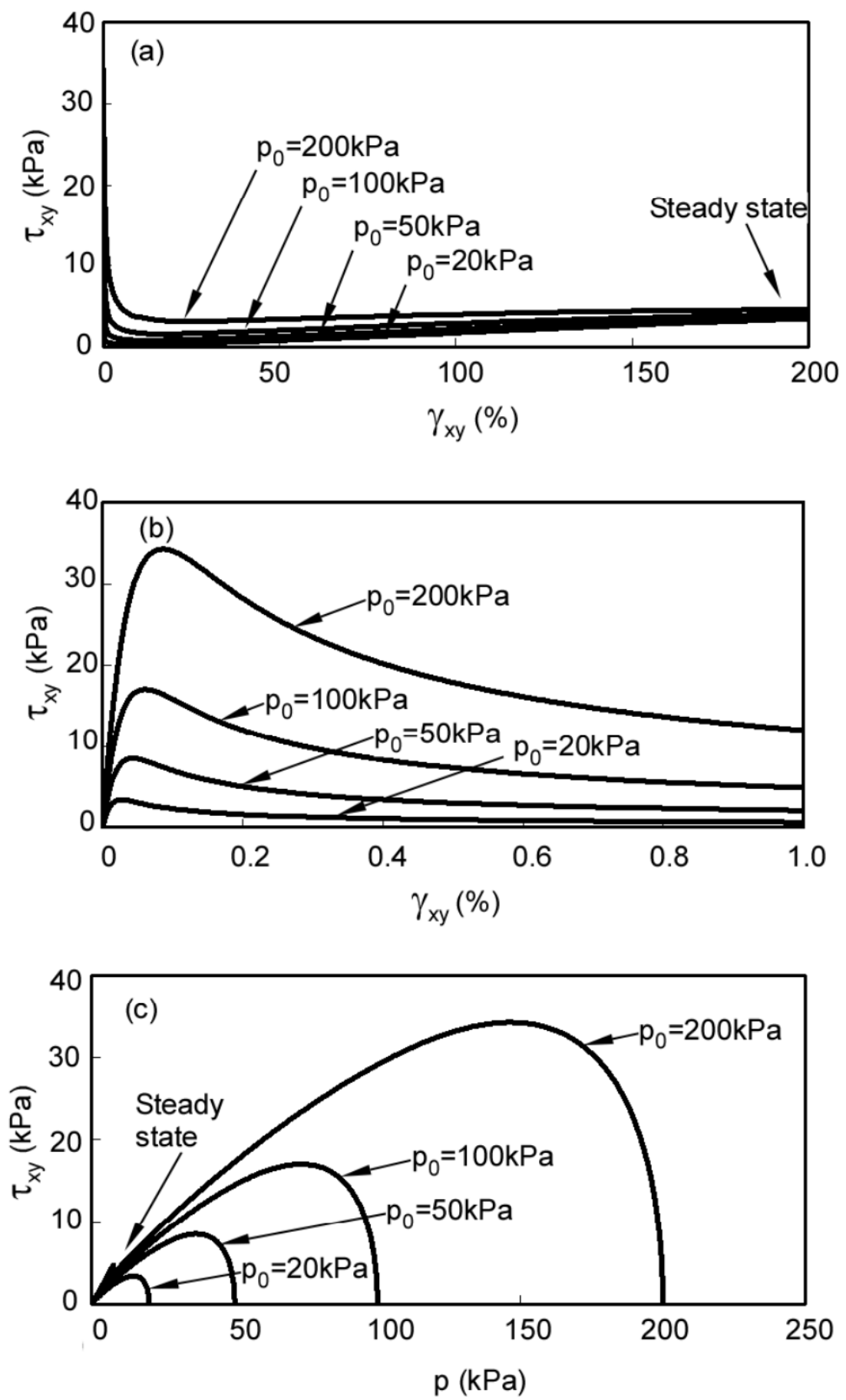

Figure 12. Undrained behavior under monotonic loading $\left(q_{\mathrm{us}}=5 \mathrm{kPa}\right)$ : (a) overall stress-strain relationship; (b) stress-strain relationship for small strain range; (c) stress path. 

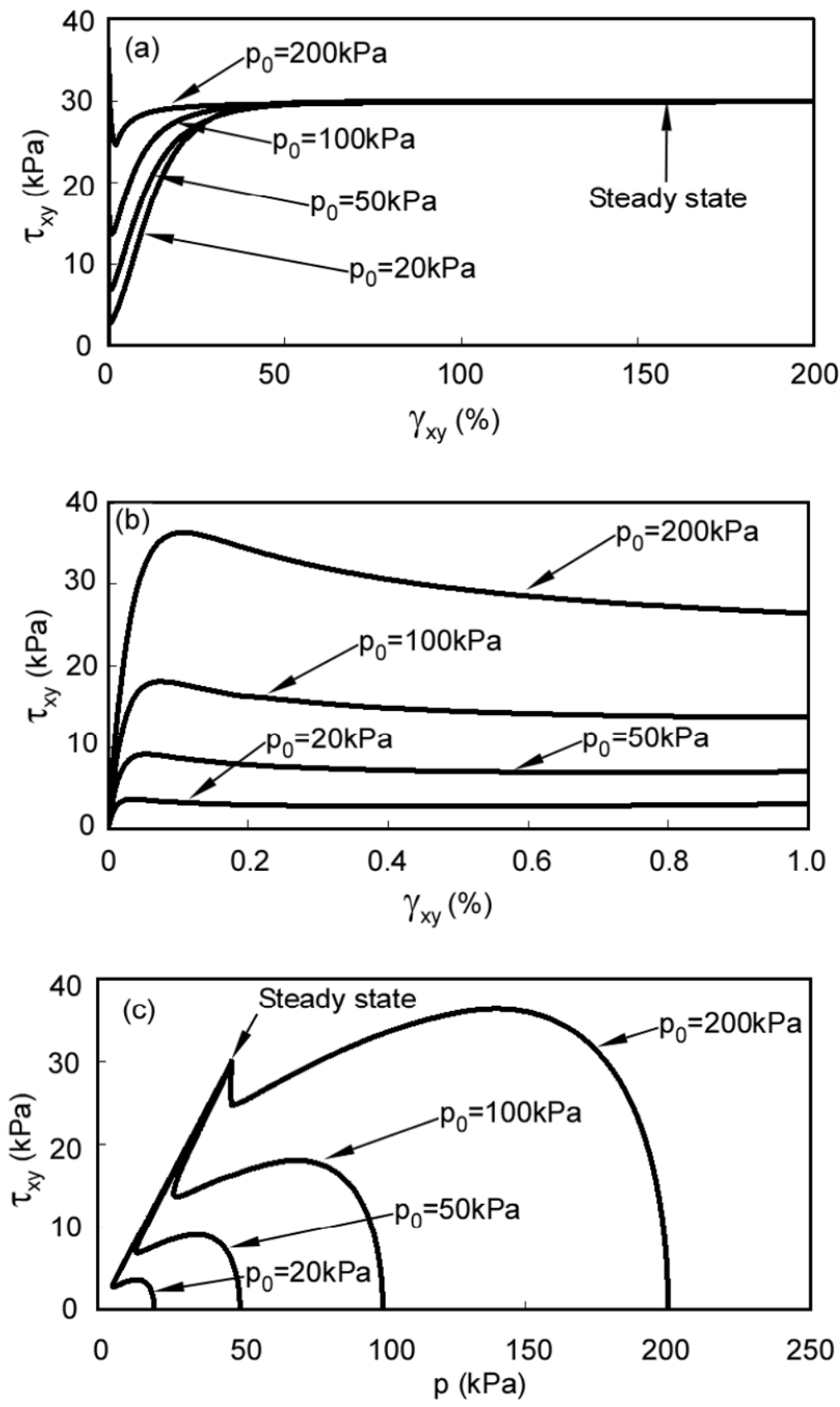

Figure 13. Undrained behavior under monotonic loading ( $\left.q_{\mathrm{us}}=30 \mathrm{kPa}\right)$ : (a) overall stress-strain relationship; (b) stress-strain relationship for small strain range; (c) stress path. 

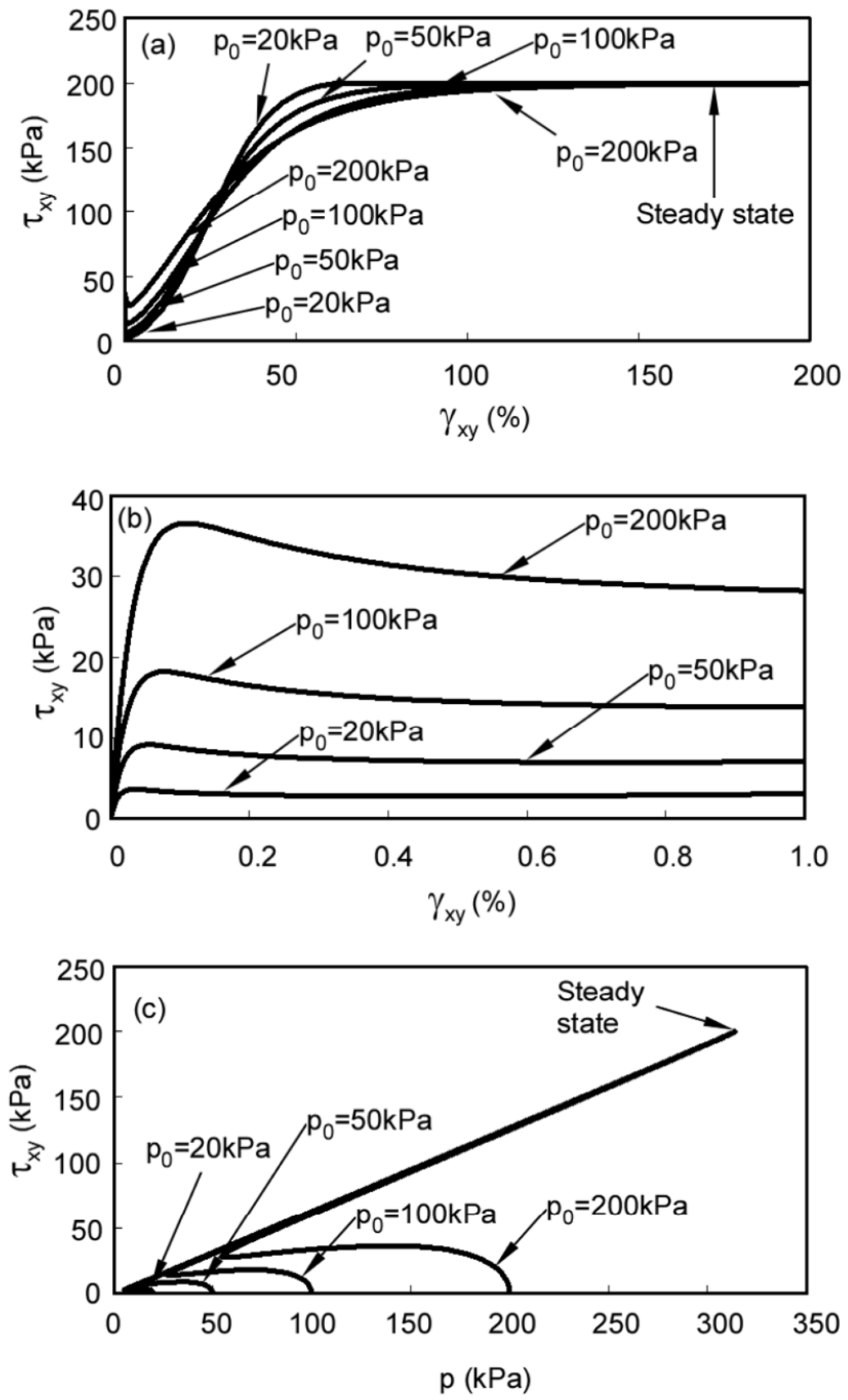

Figure 14. Undrained behavior under monotonic loading ( $\left.q_{\text {us }}=200 \mathrm{kPa}\right)$ : (a) overall stress-strain relationship; (b) stress-strain relationship for small strain range; (c) stress path. 

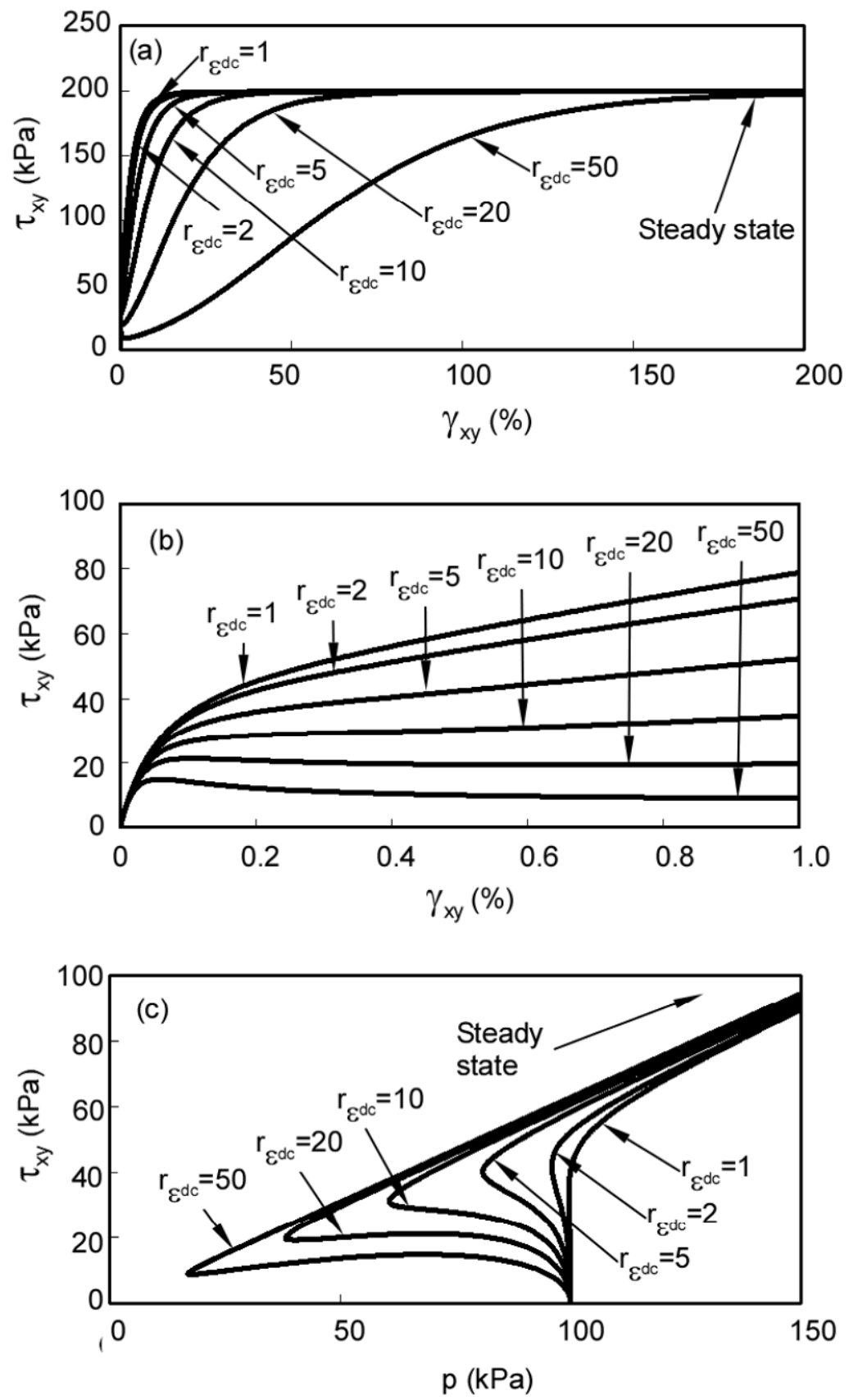

Figure 15. Undrained behavior under monotonic loading (with various $r_{\varepsilon_{\mathrm{d}}^{\mathrm{c}}}$ ): (a) overall stress-strain relationship; (b) stress-strain relationship for small strain range; (c) stress path. 

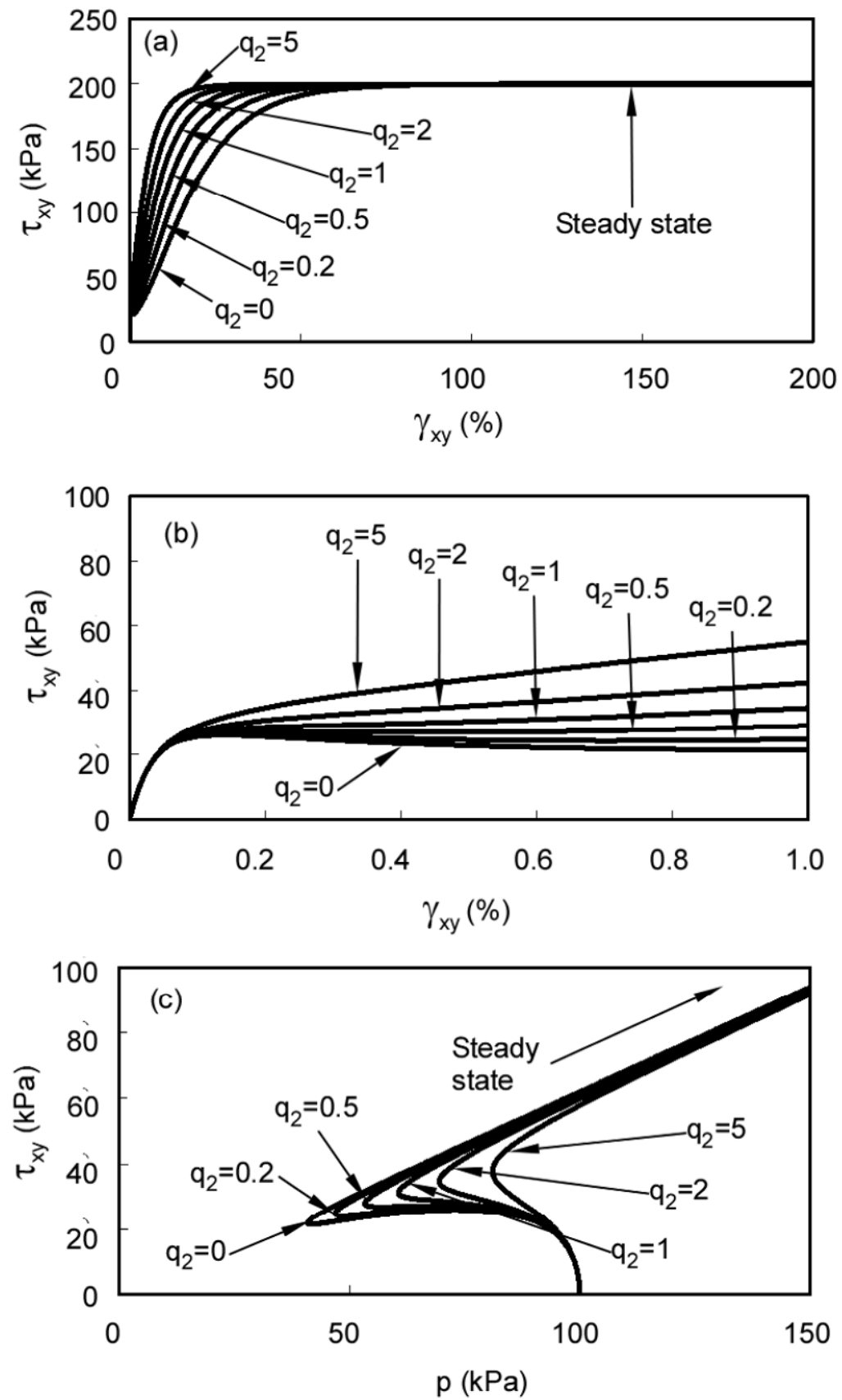

Figure 16. Undrained behavior under monotonic loading (with various $q_{2}$ ): (a) overall stress-strain relationship; (b) stress-strain relationship for small strain range; (c) stress path. 

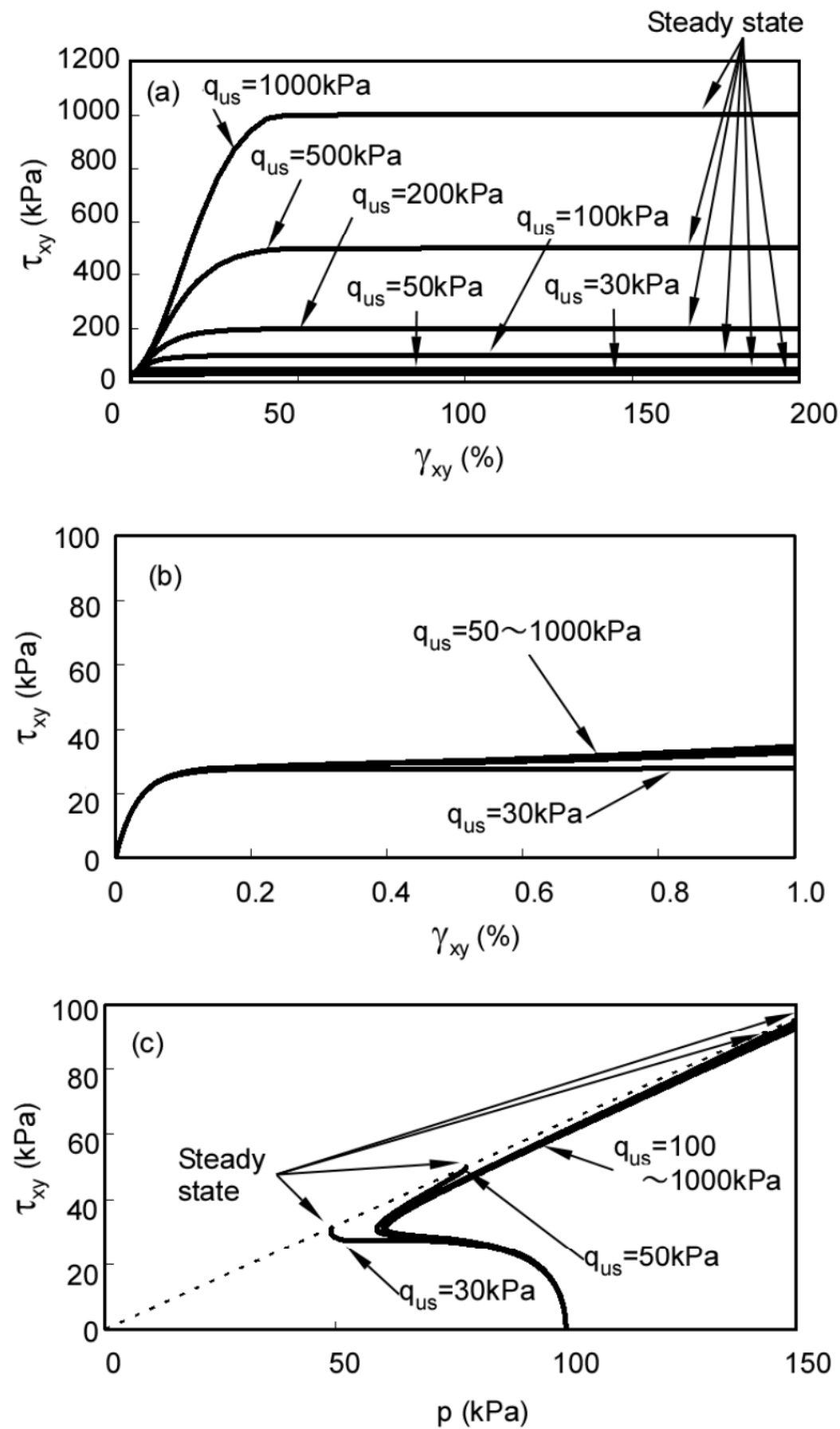

Figure 17. Undrained behavior under monotonic loading (with various $q_{\mathrm{us}}$ ): (a) overall stress-strain relationship; (b) stress-strain relationship for small strain range; (c) stress path. 

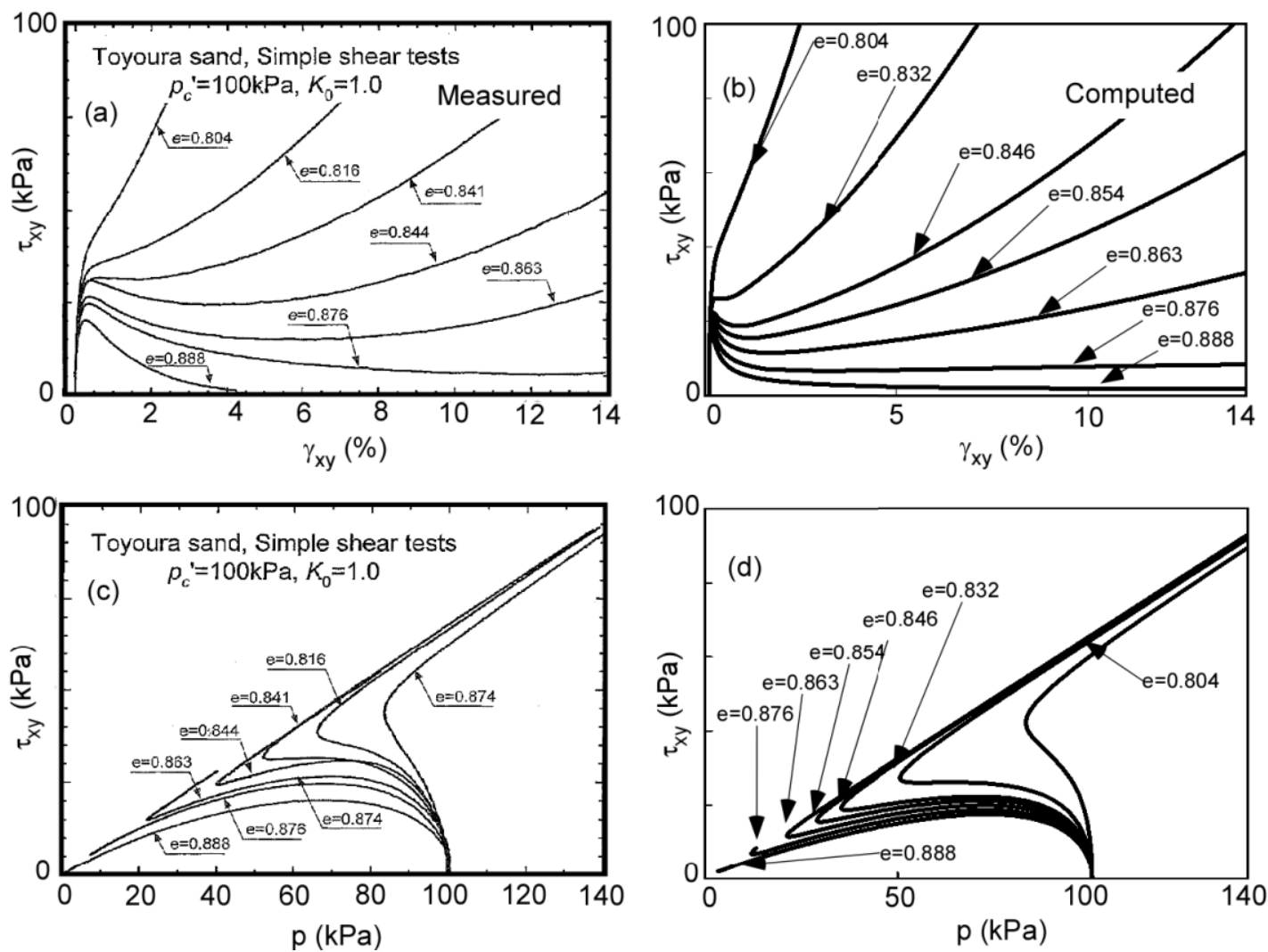

Figure 18. Undrained behavior under monotonic loading (with various void ratios) (measured data after Yoshimine et al. [46]); stress-strain relationship (measured (a), computed (b)); stress path (measured (c), computed (d)). 

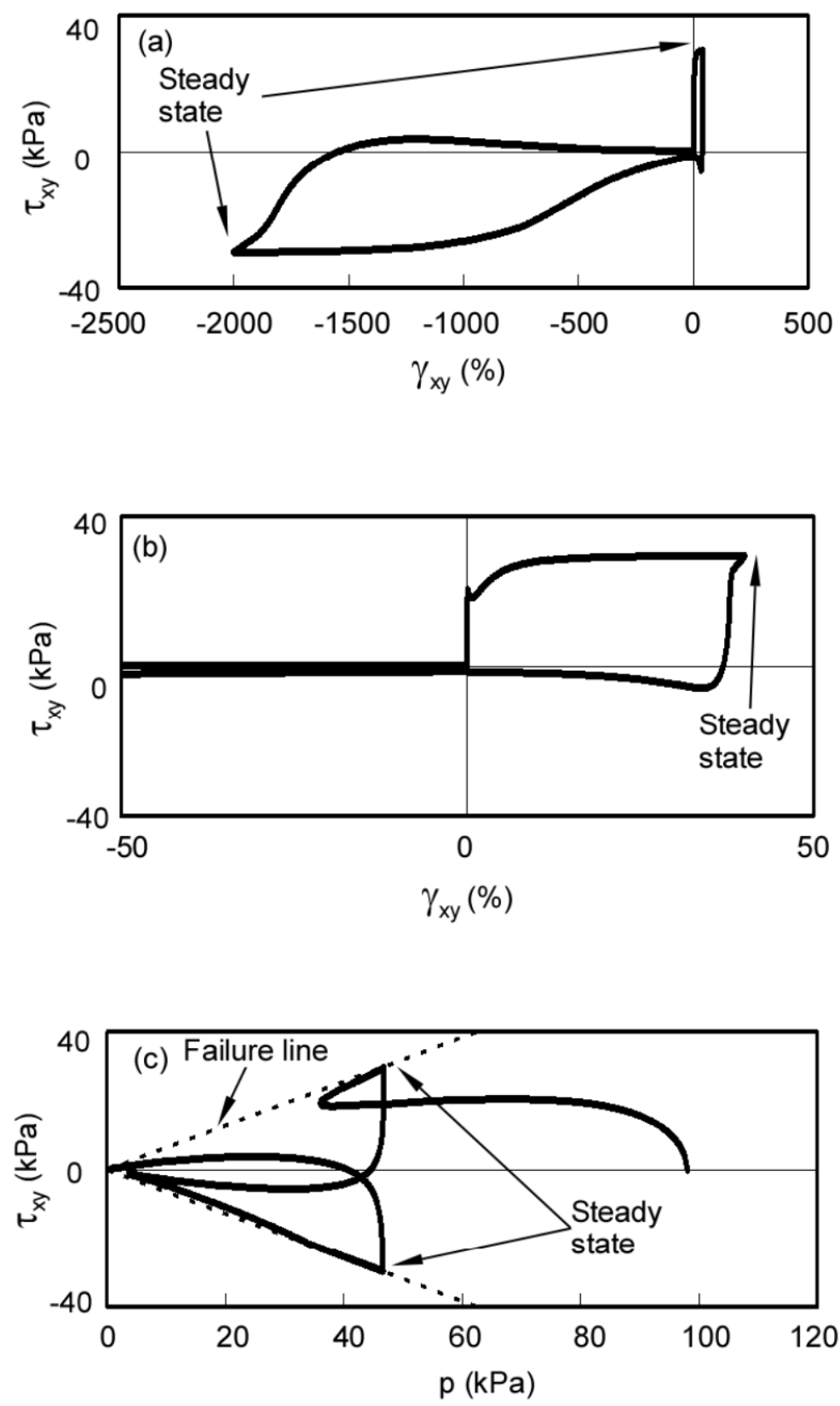

Figure 19. Undrained behavior under monotonic loading with strain reversal on both ends: (a) overall stress-strain relationship; (b) stress-strain relationship for initial half cycle; (c) stress path. 

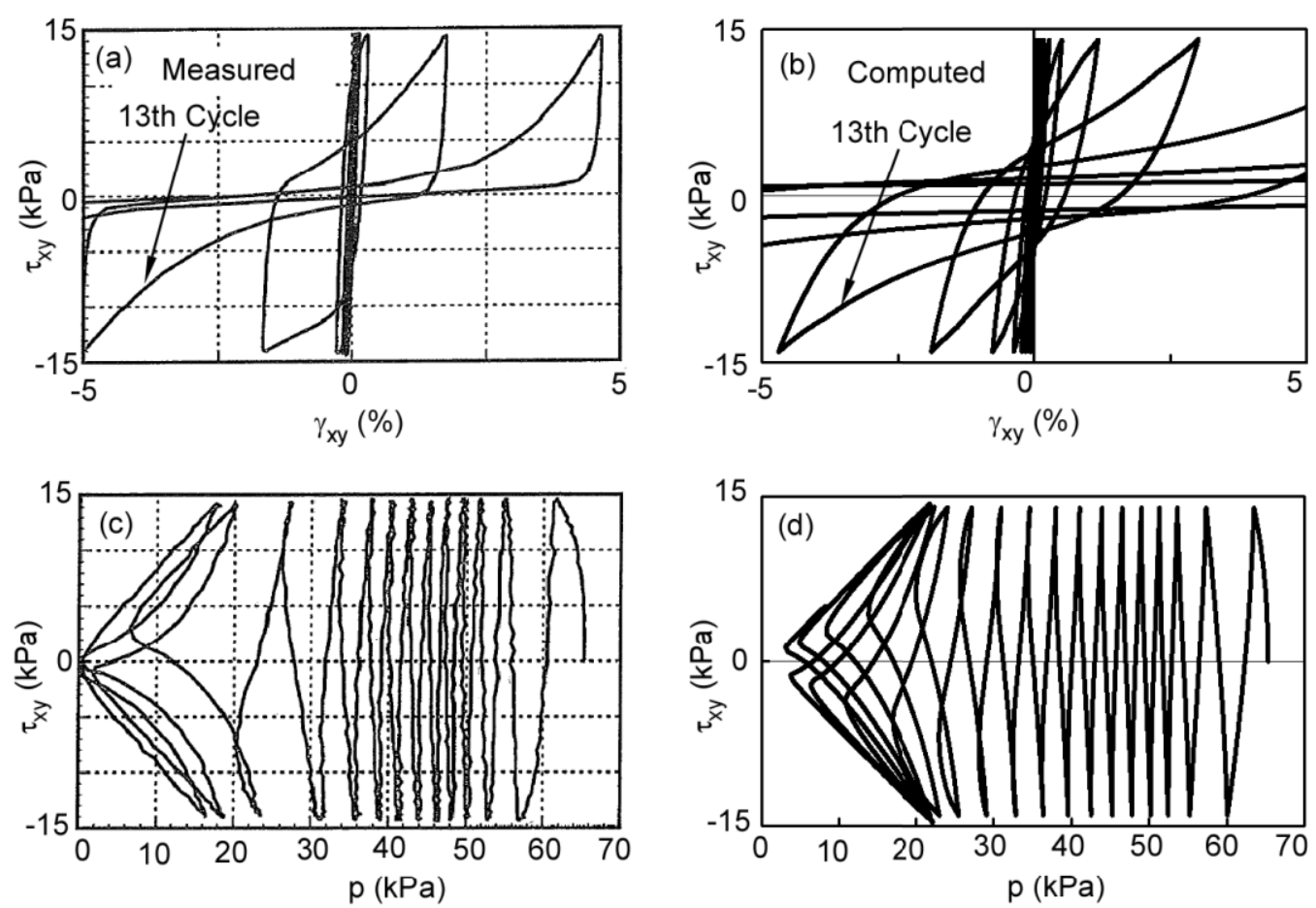

Figure 20. Cyclic undrained behavior (measured data after Matsuo et al. [47]; stress-strain relationship (measured (a), computed (b)); stress path (measured (c), computed (d)).

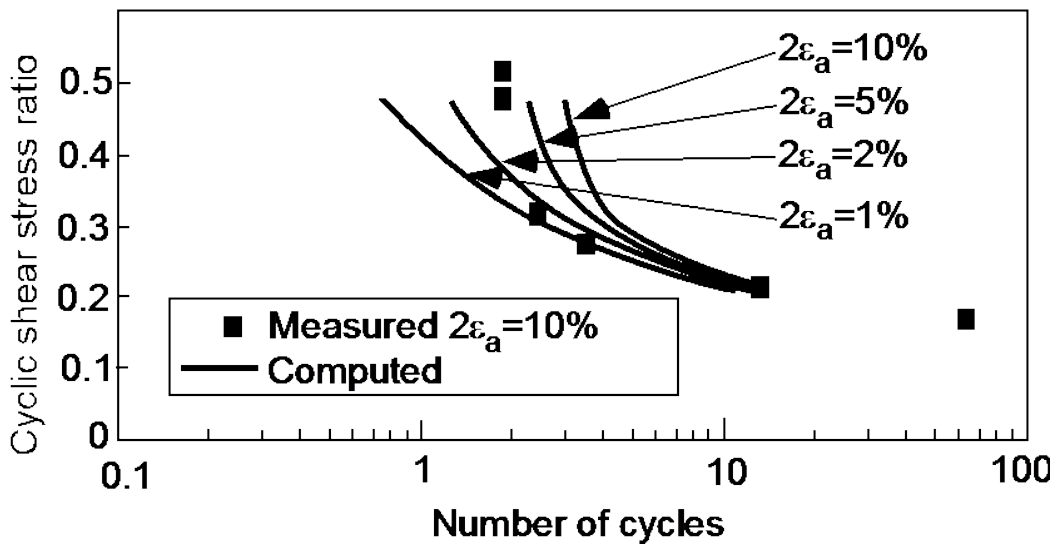

Figure 21. Computed and measured cyclic shear stress ratios (Measured data after [47]). 

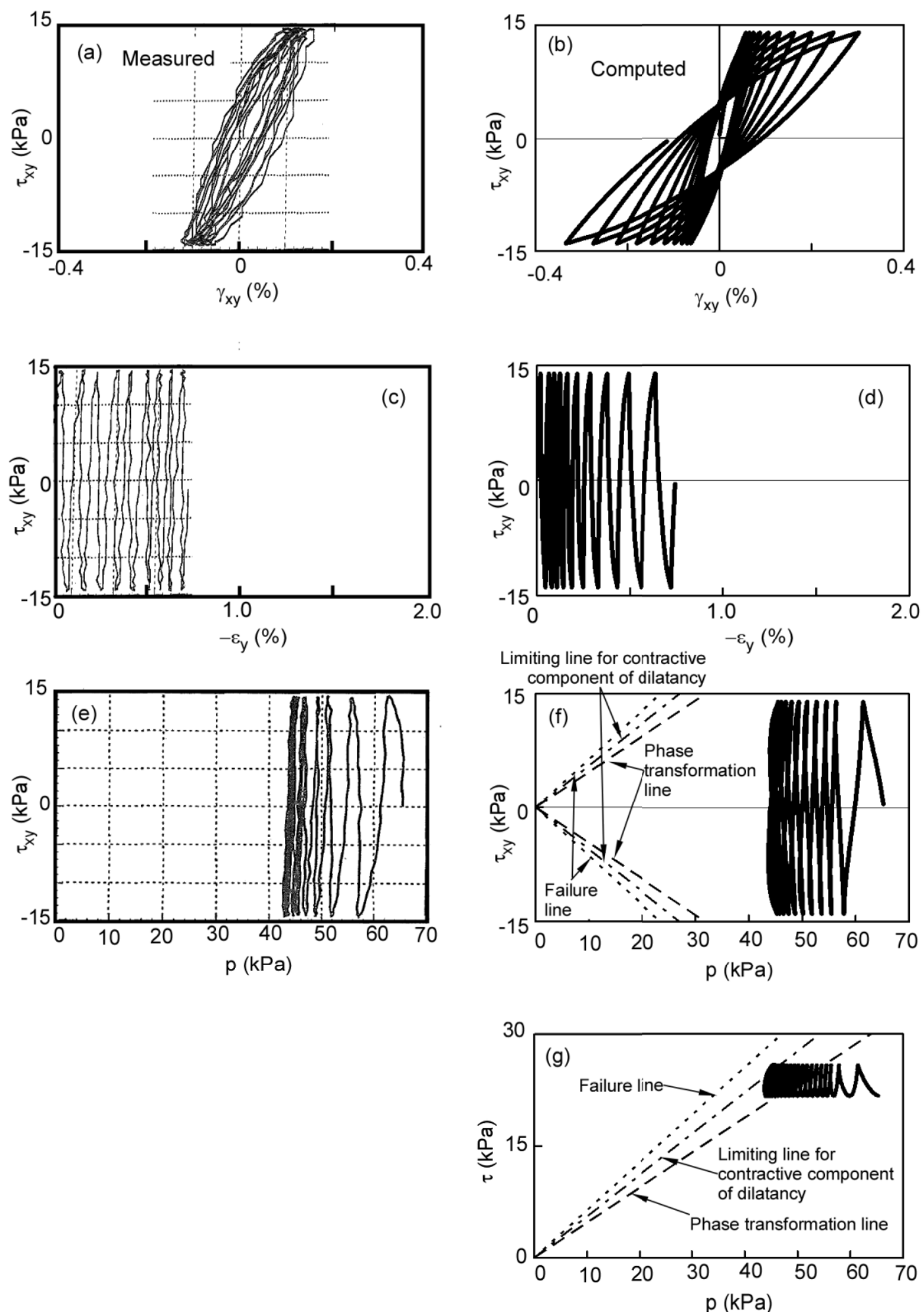

Figure 22. Cyclic undrained behavior with a constant axial stress difference (measured data after [47]); shear stress-shear strain relationship (measured (a), computed (b)); shear stress-axial strain difference relationship (measured (c), computed (d)); stress path in $\tau_{x y}-p$ plane (measured (e), computed (f)); computed stress path in $\tau-p$ plane (g). 